Applied Mathematical Sciences, Vol. 7, 2013, no. 119, 5901 - 5925

HIKARI Ltd, www.m-hikari.com

http://dx.doi.org/10.12988/ams.2013.37361

\title{
Inverse Optimal Filtering of \\ Linear Distributed Parameter Systems
}

\author{
K.D. Do \\ Department of Mechanical Engineering \\ Curtin University, Perth, WA 6845, Australia
}

Copyright (C) 2013 K.D. Do. This is an open access article distributed under the Creative Commons Attribution License, which permits unrestricted use, distribution, and reproduction in any medium, provided the original work is properly cited.

\begin{abstract}
A constructive method is developed to design inverse optimal filters to estimate the states of a class of linear distributed parameter systems (DPSs) based on the calculus of variation approach. Inverse optimality guarantees that the cost functional to be minimized is meaningful in the sense that the symmetric and positive definite weighting kernel matrix on the states is chosen after the filter design instead of being specified at the start of the filter design. Inverse optimal design enables that the Riccati nonlinear partial differential equation (PDE) can be simplified to a Bernoulli PDE, which can be solved analytically. The filter design is based on a new Green matrix formula, a new unique and bounded solution of a linear PDE, and analytical solution of a Bernoulli PDE. The inverse optimal filter design is first developed for the case where the measurements are spatially available, then is extended to the practical case where only a finite number of measurements is available.
\end{abstract}

Keywords: Distributed parameter systems, Inverse optimal filter, Bernoulli PDE, Riccati PDE

\section{Introduction}

In many physical processes, the dynamical system one wishes to estimate is described by PDEs such as chemical reactors, heat exchangers, transmission lines, vibrating beams and electrical, optical or acoustic waves. All of these are DPSs, which may be subject to bounded disturbances, and the problem of estimating the state from noisy observations is an important engineering one. The optimal filtering design techniques of these systems can be roughly classified into two main approaches.

The first approach referred to as the model approach, see for example [19], [15], [17], [6], to this problem is to obtain an approximate lumped parameter model for 
a DPS and then to apply the well-known finite-dimensional techniques [1], [8], [10]. The modal approach can only observe a finite number of modes of a DPS and has a significant drawback of computing appropriate gain matrices.

The second approach applies semigroup theory to represent PDEs as ODEs in Hilbert spaces. From here the classical optimal filtering results are extended into infinite-dimensional systems [22], [3], [11], [2]. This approach eventually results in operator Riccati equations which have similarities to the results presented here. The operator Riccati equations are nonlinear and are to be solved backward in time. These operator Riccati equations are equivalent to Riccati nonlinear PDEs in the Euclidean space. Solutions to the Riccati nonlinear PDEs are extremely rare and are only available for some very simple systems such as in [14], [20], and approximate solutions by an eigenfuncion expansion in [18], [21]. The eigenfunction expansion is usually appropriate for linear PDEs but not for nonlinear PDEs like the Riccati nonlinear PDEs. Moreover, there have no formal proof of existence and uniqueness of the symmetric and positive definite solution of these Riccati nonlinear PDEs for a sufficiently large class of linear DPSs, which cover the important practical processes.

Difficulties arisen in solving the Riccati nonlinear PDEs and two-point-boundary value problems, which are resulted from the classical design of optimal filters for distributed parameter systems, motivate the approach of designing inverse optimal filters in this paper. The difference between the direct and the inverse optimal filtering problems is that the former designs a filter that minimizes a given cost, while the latter seeks a filter that minimizes a "meaningful" cost functional, which is a part of the solution of the Bernoulli PDE. The proposed development of the inverse optimal filter design in this paper is related to the development of inverse optimal controllers for systems governed by nonlinear ODEs in [12], [9]. The inverse approach in [12], [9] uses a control Lyapunov function, which is a solution of Hamilton-JacobiBellman with a meaningful cost, for systems governed by nonlinear ODEs obtained by solving a stabilization problem.

The rest of the paper is organized as follows. The inverse optimal filtering problem is formulated in Section 2. In Section 3, we derive preliminary results that will be used in the filter design. The calculus of variation approach is utilized to derive the inverse optimal filters in Section 4. Section 5 presents an extension of the filter designed in Section 4 to the practical case where only a finite number of measurements is available. Proof of results are given in Appendices A, B, C, and D.

Notations: For a $r \times r$ positive definite matrix $\boldsymbol{A}(\boldsymbol{x}, \boldsymbol{y})$, the notation $\boldsymbol{A}^{+}(\boldsymbol{x}, \boldsymbol{y})$ denotes its generalized inverse such that $\int_{D} \boldsymbol{A}(\boldsymbol{x}, \boldsymbol{y}) \boldsymbol{A}^{+}\left(\boldsymbol{y}, \boldsymbol{x}^{\prime}\right) d \boldsymbol{y}=\boldsymbol{I} \delta\left(\boldsymbol{x}-\boldsymbol{x}^{\prime}\right)$ with $\boldsymbol{I}$ being the $r \times r$ identity matrix, and $\delta\left(\boldsymbol{x}-\boldsymbol{x}^{\prime}\right)$ being the Dirac delta function of $\left(\boldsymbol{x}-\boldsymbol{x}^{\prime}\right)$. For two vectors $\boldsymbol{a}$ and $\boldsymbol{b}$ with the same size, the notation $\left.<\boldsymbol{a}, \boldsymbol{b}\right\rangle$ denotes their inner product. For a matrix operator $\boldsymbol{A}_{\boldsymbol{x}}$, the notation $\boldsymbol{A}_{\boldsymbol{x}}^{*}$ denotes its adjoint.

\section{Problem formulation}

Let $D$ be a open bounded set in Euclidean $n$-space $E^{n}$ with piecewise smooth boundary $S$, and let $t$ denote time defined on an interval $T=\left[t_{0}, t_{f}\right]$ with $t_{f}>t_{0}$. In this 
paper, we consider a class of DPSs governed by the following linear PDE:

$$
\begin{aligned}
& \frac{\partial \boldsymbol{\chi}(\boldsymbol{x}, t)}{\partial t}=\boldsymbol{A}_{\boldsymbol{x}} \boldsymbol{\chi}(\boldsymbol{x}, t)+\boldsymbol{w}_{d}(\boldsymbol{x}, t), \quad \forall \boldsymbol{x} \in D, \\
& \boldsymbol{\chi}\left(\boldsymbol{x}, t_{0}\right)=\boldsymbol{\chi}_{0}(\boldsymbol{x})+\boldsymbol{w}_{0}(\boldsymbol{x}), \quad \forall \boldsymbol{x} \in D, \\
& \boldsymbol{\beta}_{\boldsymbol{\xi}} \boldsymbol{\chi}(\boldsymbol{\xi}, t)=\boldsymbol{w}_{b}(\boldsymbol{\xi}, t), \quad \forall \boldsymbol{\xi} \in S,
\end{aligned}
$$

where $\boldsymbol{x}=\operatorname{col}\left(x_{1}, \ldots, x_{n}\right) \in D$ is the $n$-dimensional spatial coordinate vector; $\boldsymbol{\chi}(\boldsymbol{x}, t)=$ $\operatorname{col}\left(\chi_{1}(\boldsymbol{x}, t), \ldots, \chi_{r}(\boldsymbol{x}, t)\right)$ is the $r$-dimensional vector state; $\boldsymbol{w}_{d}(\boldsymbol{x}, t)$ and $\boldsymbol{w}_{0}(\boldsymbol{x})$ are $r$ dimensional bounded disturbance vectors distributed over the interior; and $\boldsymbol{w}_{b}(\boldsymbol{\xi}, t)$ is a $r$-dimensional bounded disturbance vector distributed over the boundary.

We assume in addition that the $m_{d}$-dimensional measurement vector $\boldsymbol{z}_{d}(\boldsymbol{x}, t)$ distributed over the interior and the $m_{b}$-dimensional measurement vector $\boldsymbol{z}_{b}(\boldsymbol{x}, t)$ distributed over the boundary are available in the form

$$
\begin{aligned}
& \boldsymbol{z}_{d}(\boldsymbol{x}, t)=\boldsymbol{H}_{d}(\boldsymbol{x}, t) \boldsymbol{\chi}(\boldsymbol{x}, t)+\boldsymbol{\varepsilon}_{d}(\boldsymbol{x}, t), \\
& \boldsymbol{z}_{b}(\boldsymbol{\xi}, t)=\boldsymbol{H}_{b}(\boldsymbol{\xi}, t) \boldsymbol{\chi}(\boldsymbol{\xi}, t)+\boldsymbol{\varepsilon}_{b}(\boldsymbol{\xi}, t),
\end{aligned}
$$

where $\boldsymbol{H}_{d}(\boldsymbol{x}, t)$ is a $m_{d} \times r$ matrix defined for all $\boldsymbol{x} \in D$ and $t \in T, \boldsymbol{H}_{b}(\boldsymbol{\xi}, t)$ is a $m_{b} \times r$ matrix defined for all $\boldsymbol{\xi} \in S$ and $t \in T$, and $\boldsymbol{\varepsilon}_{d}(\boldsymbol{x}, t)$ and $\boldsymbol{\varepsilon}_{b}(\boldsymbol{\xi}, t)$ are the $m_{d}$-dimensional and $m_{b}$-dimensional vectors of bounded measurement disturbances, respectively. In this paper, we impose the following assumption.

\section{Assumption 2.1}

1. The matrix operators $\boldsymbol{A}_{\boldsymbol{x}}$ and $\boldsymbol{\beta}_{\boldsymbol{\xi}}$ are given by

$$
\begin{aligned}
& \boldsymbol{A}_{\boldsymbol{x}}[\bullet]=\sum_{i, j=1}^{n} \boldsymbol{A}_{i j}(\boldsymbol{x}, t) \frac{\partial^{2}[\bullet]}{\partial x_{i} \partial x_{j}}+\sum_{i=1}^{n} \boldsymbol{B}_{i}(\boldsymbol{x}, t) \frac{\partial[\bullet]}{\partial x_{i}}+\boldsymbol{C}(\boldsymbol{x}, t)[\bullet], \\
& \boldsymbol{\beta}_{\boldsymbol{\xi}}[\bullet]=\sum_{j=1}^{n} \boldsymbol{A}_{j}(\boldsymbol{\xi}, t) \frac{\partial[\bullet]}{\partial x_{j}}+\boldsymbol{F}(\boldsymbol{\xi}, t)[\bullet]
\end{aligned}
$$

where the $\boldsymbol{A}_{i j}(\boldsymbol{x}, t), \boldsymbol{B}_{i}(\boldsymbol{x}, t), \boldsymbol{C}(\boldsymbol{x}, t)$, and $\boldsymbol{F}(\boldsymbol{\xi}, t)$ are $r \times r$ matrices, and

$$
\boldsymbol{A}_{j}(\boldsymbol{\xi}, t)=\sum_{i=1}^{n} \boldsymbol{A}_{i j}(\boldsymbol{\xi}, t) \cos \left(\boldsymbol{n}_{\boldsymbol{\xi}}, x_{i}\right),
$$

with $\boldsymbol{n}_{\boldsymbol{\xi}}$ being the outward normal to the boundary $S$ at the point $\boldsymbol{\xi} \in S$, and $\left(\boldsymbol{n}_{\boldsymbol{\xi}}, x_{i}\right)$ being the angle between the outward normal $\boldsymbol{n}_{\boldsymbol{\xi}}$ and the $x_{i}$-axis. Furthermore, the matrix $\boldsymbol{A}_{i j}(\boldsymbol{x}, t)$ is symmetric, i.e., $\boldsymbol{A}_{i j}(\boldsymbol{x}, t)=\boldsymbol{A}_{j i}(\boldsymbol{x}, t)$. In the second equation of (3), we have denoted the notation $\frac{\partial[\bullet]}{\partial x_{j}}=\left.\frac{\partial[\bullet(x, t)]}{\partial x_{j}}\right|_{\boldsymbol{x}=\boldsymbol{\xi}}$.

2. There exist symmetric and positive definite matrices $\boldsymbol{Q}_{d}^{+}(\boldsymbol{x}, \boldsymbol{y}, t)$ and $\boldsymbol{Q}_{b}^{+}(\boldsymbol{\xi}, \boldsymbol{\alpha}, t)$ such that the matrices $\overline{\boldsymbol{Q}}_{d}(\boldsymbol{x}, \boldsymbol{y}, t)$ and $\overline{\boldsymbol{Q}}_{b}(\boldsymbol{\xi}, \boldsymbol{\alpha}, t)$ defined by

$$
\begin{aligned}
\overline{\boldsymbol{Q}}_{d}(\boldsymbol{x}, \boldsymbol{y}, t) & =\boldsymbol{H}_{d}^{T}(\boldsymbol{x}, t) \boldsymbol{Q}_{d}^{+}(\boldsymbol{x}, \boldsymbol{y}, t) \boldsymbol{H}_{d}(\boldsymbol{y}, t), \\
\overline{\boldsymbol{Q}}_{b}(\boldsymbol{\xi}, \boldsymbol{\alpha}, t) & =\boldsymbol{H}_{b}^{T}(\boldsymbol{\xi}, t) \boldsymbol{Q}_{b}^{+}(\boldsymbol{\xi}, \boldsymbol{\alpha}, t) \boldsymbol{H}_{b}(\boldsymbol{\alpha}, t)
\end{aligned}
$$

are bounded and symmetric and positive definite. 
3. Let the boundary $S$ be represented locally by the equation

$$
\operatorname{col}\left(\psi_{1}(\boldsymbol{x}), \ldots, \psi_{r}(\boldsymbol{x})\right)=0
$$

in the neighborhood of the point $\boldsymbol{\xi} \in S$ and let $\boldsymbol{\psi}_{i}(\boldsymbol{x}) \geq 0, i=1, \ldots, r$, if $\boldsymbol{x} \in \bar{D}$ where $\bar{D}$ is the closure of $D$, i.e., $\bar{D}=D \cup S$. Moreover, let $\delta\left(\psi_{i}(\boldsymbol{x})\right)$ be a generalized function concentrated on the boundary $S$, an $(n-1)$-dimensional manifold [7]. Then we have the formula [7]

$$
\int_{D} \boldsymbol{\delta}(\boldsymbol{\psi}(\boldsymbol{x})) \boldsymbol{\phi}(\boldsymbol{x}) d \boldsymbol{x}=\int_{S} \boldsymbol{\phi}(\boldsymbol{\xi}) d S_{\boldsymbol{\xi}},
$$

where $\boldsymbol{\delta}(\boldsymbol{\psi}(\boldsymbol{x}))=\operatorname{diag}\left(\delta\left(\psi_{1}(\boldsymbol{x})\right), \ldots, \delta\left(\psi_{r}(\boldsymbol{x})\right)\right)$. There exist symmetric and positive definite matrices $\boldsymbol{L}_{d}(\boldsymbol{x}, \boldsymbol{y}, t)$ and $\boldsymbol{L}_{b}(\boldsymbol{\xi}, t)$ such that the system

$$
\begin{aligned}
& \frac{\partial \boldsymbol{Z}(\boldsymbol{x}, \boldsymbol{y}, t)}{\partial t}=-\boldsymbol{Z}(\boldsymbol{x}, \boldsymbol{y}, t) \overline{\boldsymbol{A}}_{\boldsymbol{y}}-\left[\overline{\boldsymbol{A}}_{\boldsymbol{x}}\right]^{T} \boldsymbol{Z}(\boldsymbol{x}, \boldsymbol{y}, t), \\
& \boldsymbol{Z}\left(\boldsymbol{x}, \boldsymbol{y}, t_{0}\right)=\boldsymbol{Z}_{0}(\boldsymbol{x}, \boldsymbol{y}), \\
& \overline{\boldsymbol{\beta}}_{\boldsymbol{\xi}} \boldsymbol{Z}(\boldsymbol{\xi}, \boldsymbol{y}, t)=0
\end{aligned}
$$

is exponentially stable at the origin, where

$$
\begin{aligned}
& \overline{\boldsymbol{A}}_{\boldsymbol{x}}[\bullet]=\boldsymbol{A}_{\boldsymbol{x}}[\bullet]+\boldsymbol{L}_{d}(\boldsymbol{x}, \boldsymbol{y}, t)[\bullet], \\
& \overline{\boldsymbol{\beta}}_{\boldsymbol{\xi}}[\bullet]=\boldsymbol{\beta}_{\boldsymbol{\xi}}[\bullet]-\boldsymbol{\delta}(\boldsymbol{\psi}(\boldsymbol{x})) \boldsymbol{L}_{b}(\boldsymbol{\xi}, t)[\bullet]
\end{aligned}
$$

In this paper, we consider the following filter design objective.

Filter Design Objective 2.1 Subject to the constraints defined by (1), design an estimate $\hat{\boldsymbol{\chi}}(\boldsymbol{x}, t)$ for $\boldsymbol{\chi}(\boldsymbol{x}, t)$ so as to minimize the following cost functional:

$$
J=\int_{t_{0}}^{t_{f}} L d t+J_{0}
$$

where

$$
\begin{aligned}
L & =\frac{1}{2} \int_{D^{2}}\left\langle\left(\frac{\partial \boldsymbol{\chi}(\boldsymbol{x}, t)}{\partial t}-\boldsymbol{A}_{\boldsymbol{x}} \boldsymbol{\chi}(\boldsymbol{x}, t)\right), \boldsymbol{R}_{d}^{+}(\boldsymbol{x}, \boldsymbol{y}, t)\left(\frac{\partial \boldsymbol{\chi}(\boldsymbol{y}, t)}{\partial t}-\boldsymbol{A}_{\boldsymbol{y}} \chi(\boldsymbol{y}, t)\right)\right\rangle d \boldsymbol{x} d \boldsymbol{y} \\
& +\frac{1}{2} \int_{S^{2}}\left\langle\boldsymbol{\beta}_{\boldsymbol{\xi}} \boldsymbol{\chi}(\boldsymbol{\xi}, t), \boldsymbol{R}_{b}^{+}(\boldsymbol{\xi}, \boldsymbol{\alpha}, t) \boldsymbol{\beta}_{\boldsymbol{\alpha}} \boldsymbol{\chi}(\boldsymbol{\alpha}, t)\right\rangle d S_{\boldsymbol{\xi}} d S_{\boldsymbol{\alpha}} \\
& +\frac{1}{2} \int_{D^{2}}\left\langle\left[\boldsymbol{z}_{d}(\boldsymbol{x}, t)-\boldsymbol{H}_{d}(\boldsymbol{x}, t) \boldsymbol{\chi}(\boldsymbol{x}, t)\right], \boldsymbol{Q}_{d}^{+}(\boldsymbol{x}, \boldsymbol{y}, t)\left[\boldsymbol{z}_{d}(\boldsymbol{y}, t)-\boldsymbol{H}_{d}(\boldsymbol{y}, t) \boldsymbol{\chi}(\boldsymbol{y}, t)\right]\right\rangle d \boldsymbol{x} d \boldsymbol{y} \\
& +\frac{1}{2} \int_{S^{2}}\left\langle\left[\boldsymbol{z}_{b}(\boldsymbol{\xi}, t)-\boldsymbol{H}_{b}(\boldsymbol{\xi}, t) \boldsymbol{\chi}(\boldsymbol{\xi}, t)\right], \boldsymbol{Q}_{b}^{+}(\boldsymbol{\xi}, \boldsymbol{\alpha}, t)\left[\boldsymbol{z}_{b}(\boldsymbol{\alpha}, t)-\boldsymbol{H}_{b}(\boldsymbol{\alpha}, t) \boldsymbol{\chi}(\boldsymbol{\alpha}, t)\right]\right\rangle d S_{\boldsymbol{\xi}} d S_{\boldsymbol{\alpha}}, \\
J_{0} & =\frac{1}{2} \int_{D^{2}}\left\langle\left[\chi\left(\boldsymbol{x}, t_{0}\right)-\chi_{0}(\boldsymbol{x})\right], \boldsymbol{P}_{0}^{+}(\boldsymbol{x}, \boldsymbol{y})\left[\boldsymbol{\chi}(\boldsymbol{y}, t)-\boldsymbol{\chi}_{0}(\boldsymbol{y})\right]\right\rangle d \boldsymbol{x} d \boldsymbol{y},
\end{aligned}
$$

with the symmetric and positive definite matrices $\boldsymbol{Q}_{d}(\boldsymbol{x}, \boldsymbol{y}, t)$ and $\boldsymbol{Q}_{b}(\boldsymbol{\xi}, \boldsymbol{\alpha}, t)$ being defined in Item 2) of Assumption 2.1, and $\boldsymbol{P}_{0}(\boldsymbol{x}, \boldsymbol{y}), \boldsymbol{R}_{d}(\boldsymbol{x}, \boldsymbol{y}, t)$ and $\boldsymbol{R}_{b}(\boldsymbol{\xi}, \boldsymbol{\alpha}, t)$ being symmetric and positive definite matrices. 


\section{Preliminaries}

In this section, we derive a matrix Green's formula, a formula for differentiation of a generalized inverse matrix, a derivation of the unique and bounded solution of a linear PDE, and an analytical solution of a Bernoulli nonlinear PDE. These results will be used in the filter design.

\subsection{Matrix Green's Formula}

Lemma 3.1 Consider the matrix differential operators $\boldsymbol{A}_{\boldsymbol{x}}$ and $\boldsymbol{\beta}_{\boldsymbol{\xi}}$ defined in (3). Then

$$
\begin{aligned}
\int_{D}\left[\left\langle\boldsymbol{A}_{\boldsymbol{x}} \gamma(\boldsymbol{x}, t), \boldsymbol{\lambda}(\boldsymbol{x}, t)\right\rangle\right. & \left.-\left\langle\boldsymbol{\gamma}(\boldsymbol{x}, t), \boldsymbol{A}_{\boldsymbol{x}}^{*} \boldsymbol{\lambda}(\boldsymbol{x}, t)\right\rangle\right] d \boldsymbol{x}= \\
& \int_{S}\left[\left\langle\boldsymbol{\beta}_{\boldsymbol{\xi}} \gamma(\boldsymbol{\xi}, t), \boldsymbol{\lambda}(\boldsymbol{\xi}, t)\right\rangle-\left\langle\gamma(\boldsymbol{\xi}, t), \boldsymbol{\beta}_{\boldsymbol{\xi}}^{*} \boldsymbol{\lambda}(\boldsymbol{\xi}, t)\right\rangle\right] d S_{\boldsymbol{\xi}},
\end{aligned}
$$

where $d \boldsymbol{x}=d x_{1} d x_{2} \ldots d x_{n}$ and $d S_{\boldsymbol{\xi}}$ is the surface element of $S$ at the point $\boldsymbol{\xi}$. The adjoint operators $\boldsymbol{A}_{\boldsymbol{x}}^{*}$ and $\boldsymbol{\beta}_{\boldsymbol{\xi}}^{*}$ are given by

$$
\begin{aligned}
& \boldsymbol{A}_{\boldsymbol{x}}^{*}[\bullet]=\sum_{i, j=1}^{n} \frac{\partial^{2}\left(\boldsymbol{A}_{i j}(\boldsymbol{x}, t)[\bullet]\right)}{\partial x_{i} \partial x_{j}}-\sum_{i=1}^{n} \frac{\partial\left(\boldsymbol{B}_{i}(\boldsymbol{x}, t)[\bullet]\right)}{\partial x_{i}}+\boldsymbol{C}(\boldsymbol{x}, t)[\bullet] \\
& \boldsymbol{\beta}_{\boldsymbol{\xi}}^{*}[\bullet]=\sum_{j=1}^{n} \boldsymbol{A}_{j}(\boldsymbol{\xi}, t) \frac{\partial[\bullet]}{\partial x_{j}}-\boldsymbol{K}(\boldsymbol{\xi}, t)[\bullet]+\boldsymbol{F}(\boldsymbol{\xi}, t)[\bullet]
\end{aligned}
$$

with

$$
\boldsymbol{K}(\boldsymbol{\xi}, t)=\sum_{i=1}^{n}\left[\boldsymbol{B}_{i}(\boldsymbol{\xi}, t)-\sum_{j=1}^{n} \frac{\partial \boldsymbol{A}_{i j}(\boldsymbol{\xi}, t)}{\partial x_{j}}\right] \cos \left(\boldsymbol{n}_{\boldsymbol{\xi}}, x_{i}\right)
$$

We have denoted the notation $\frac{\partial[\boldsymbol{\bullet}]}{\partial x_{j}}=\left.\frac{\partial[\bullet(x, t)]}{\partial x_{j}}\right|_{\boldsymbol{x}=\boldsymbol{\xi}}$ in (13) and (14).

Proof. See Appendix A.

\subsection{Time derivative of the generalized inverse of a matrix}

Lemma 3.2 Let $\boldsymbol{A}^{+}(\boldsymbol{x}, \boldsymbol{y}, t)$ denote the generalized inverse of a matrix $\boldsymbol{A}(\boldsymbol{x}, \boldsymbol{y}, t)$, i.e,

$$
\int_{D} \boldsymbol{A}\left(\boldsymbol{x}^{\prime}, \boldsymbol{y}^{\prime}, t\right) \boldsymbol{A}^{+}\left(\boldsymbol{y}^{\prime}, \boldsymbol{y}, t\right) d \boldsymbol{y}^{\prime}=\boldsymbol{I} \delta\left(\boldsymbol{x}^{\prime}-\boldsymbol{y}\right) .
$$

The time derivative of the generalized inverse of $\boldsymbol{A}^{+}(\boldsymbol{x}, \boldsymbol{y}, t)$ is given by

$$
\frac{\partial \boldsymbol{A}^{+}(\boldsymbol{x}, \boldsymbol{y}, t)}{\partial t}=-\int_{D^{2}} \boldsymbol{A}^{+}\left(\boldsymbol{x}, \boldsymbol{x}^{\prime}, t\right) \frac{\partial \boldsymbol{A}\left(\boldsymbol{x}, \boldsymbol{y}^{\prime}, t\right)}{\partial t} \boldsymbol{A}^{+}\left(\boldsymbol{y}^{\prime}, \boldsymbol{y}, t\right) d \boldsymbol{x}^{\prime} d \boldsymbol{y}^{\prime}
$$

Proof. See Appendix B. 


\subsection{Existence and uniqueness of the solution of a PDE}

Lemma 3.3 Consider the following matrix linear PDE:

$$
\begin{aligned}
& \frac{\partial \boldsymbol{N}(\boldsymbol{x}, \boldsymbol{y}, t)}{\partial t}=-\overline{\boldsymbol{A}}_{\boldsymbol{x}} \boldsymbol{N}(\boldsymbol{x}, \boldsymbol{y}, t)-\boldsymbol{N}(\boldsymbol{x}, \boldsymbol{y}, t) \overline{\boldsymbol{A}}_{\boldsymbol{y}}^{T}+\boldsymbol{R}(\boldsymbol{x}, \boldsymbol{y}, t), \\
& \boldsymbol{N}\left(\boldsymbol{x}, \boldsymbol{y}, t_{0}\right)=\boldsymbol{N}_{0}(\boldsymbol{x}, \boldsymbol{y}), \\
& \overline{\boldsymbol{\beta}}_{\boldsymbol{\xi}} \boldsymbol{N}(\boldsymbol{\xi}, \boldsymbol{y}, t)=0, \quad \boldsymbol{\xi} \in S, \boldsymbol{y} \in D,
\end{aligned}
$$

where $\overline{\boldsymbol{A}}_{\boldsymbol{x}}$ and $\overline{\boldsymbol{\beta}}_{\boldsymbol{\xi}}$ are defined in $(9), \boldsymbol{R}(\boldsymbol{x}, \boldsymbol{y}, t)$ and $\boldsymbol{N}_{0}(\boldsymbol{x}, \boldsymbol{y})$ are bounded and symmetric matrices. Then there exists a unique bounded solution $\boldsymbol{N}(\boldsymbol{x}, \boldsymbol{y}, t)$ given by

$$
\begin{aligned}
& \boldsymbol{N}(\boldsymbol{x}, \boldsymbol{y}, t)=\int_{D^{2}} \boldsymbol{G}\left(\boldsymbol{x}, t ; \boldsymbol{x}^{\prime}, t_{0}\right) \boldsymbol{N}_{0}\left(\boldsymbol{x}^{\prime}, \boldsymbol{y}^{\prime}\right) \boldsymbol{G}^{T}\left(\boldsymbol{y}, t ; \boldsymbol{y}^{\prime}, t_{0}\right) d \boldsymbol{x}^{\prime} d \boldsymbol{y}^{\prime}+ \\
& \int_{t_{0}}^{t} \int_{D^{2}} \boldsymbol{G}\left(\boldsymbol{x}, t-\tau+t_{0} ; \boldsymbol{x}^{\prime}, t_{0}\right) \boldsymbol{R}\left(\boldsymbol{x}^{\prime}, \boldsymbol{y}^{\prime}, \tau\right) \boldsymbol{G}^{T}\left(\boldsymbol{y}, t-\tau+t_{0} ; \boldsymbol{y}^{\prime}, t_{0}\right) d \boldsymbol{x}^{\prime} d \boldsymbol{y}^{\prime} d \tau,
\end{aligned}
$$

where the Green function $\boldsymbol{G}\left(\boldsymbol{x}, t ; \boldsymbol{x}^{\prime}, t_{0}\right)$ satisfies

$$
\begin{aligned}
& \frac{\partial \boldsymbol{G}\left(\boldsymbol{x}, t ; \boldsymbol{x}^{\prime}, t_{0}\right)}{\partial t}=-\overline{\boldsymbol{A}}_{\boldsymbol{x}} \boldsymbol{G}\left(\boldsymbol{x}, t ; \boldsymbol{x}^{\prime}, t_{0}\right), \\
& \boldsymbol{G}\left(\boldsymbol{x}, t_{0} ; \boldsymbol{x}^{\prime}, t_{0}\right)=\boldsymbol{I} \delta\left(\boldsymbol{x}-\boldsymbol{x}^{\prime}\right) \\
& \overline{\boldsymbol{\beta}}_{\boldsymbol{\xi}} \boldsymbol{G}\left(\boldsymbol{\xi}, t ; \boldsymbol{x}^{\prime}, t_{0}\right)=0 .
\end{aligned}
$$

Proof. See Appendix C.

\subsection{Analytical solution of a Bernoulli nonlinear PDE}

Lemma 3.4 Consider the following Bernoulli nonlinear PDE

$$
\begin{aligned}
& \frac{\partial \boldsymbol{P}(\boldsymbol{x}, \boldsymbol{y}, t)}{\partial t}=\overline{\boldsymbol{A}}_{\boldsymbol{x}} \boldsymbol{P}(\boldsymbol{x}, \boldsymbol{y}, t)-\int_{D^{2}} \boldsymbol{P}\left(\boldsymbol{x}, \boldsymbol{x}^{\prime}, t\right) \overline{\boldsymbol{R}}_{d}\left(\boldsymbol{x}^{\prime}, \boldsymbol{x}^{\prime \prime}, t\right) \boldsymbol{P}\left(\boldsymbol{x}^{\prime \prime}, \boldsymbol{y}, t\right) d \boldsymbol{x}^{\prime} d \boldsymbol{x}^{\prime \prime} \\
& \quad+\boldsymbol{P}(\boldsymbol{x}, \boldsymbol{y}, t)\left[\overline{\boldsymbol{A}}_{\boldsymbol{y}}\right]^{T}-\int_{S^{2}} \boldsymbol{P}(\boldsymbol{x}, \boldsymbol{\xi}, t) \overline{\boldsymbol{R}}_{b}(\boldsymbol{\xi}, \boldsymbol{\gamma}, t) \boldsymbol{P}(\boldsymbol{\gamma}, \boldsymbol{y}, t) d S_{\boldsymbol{\xi}} d S_{\gamma} ; \quad(\boldsymbol{x}, \boldsymbol{y}) \in D \\
& \boldsymbol{P}\left(\boldsymbol{x}, \boldsymbol{y}, t_{0}\right)=\boldsymbol{P}_{0}(\boldsymbol{x}, \boldsymbol{y}) ; \quad(\boldsymbol{x}, \boldsymbol{y}) \in D \\
& \overline{\boldsymbol{\beta}}_{\boldsymbol{\xi}} \boldsymbol{P}(\boldsymbol{\xi}, \boldsymbol{y}, t)=0 ; \quad \boldsymbol{\xi} \in S, \boldsymbol{y} \in D
\end{aligned}
$$

where $\overline{\boldsymbol{R}}_{d}(\boldsymbol{x}, \boldsymbol{y}, t), \overline{\boldsymbol{R}}_{b}(\boldsymbol{\xi}, \boldsymbol{\gamma}, t)$, and $\boldsymbol{P}_{0}(\boldsymbol{x}, \boldsymbol{y})$ are a symmetric and positive definite matrices, and $\overline{\boldsymbol{A}}_{\boldsymbol{x}}$ and $\overline{\boldsymbol{\beta}}_{\boldsymbol{\xi}}$ are defined in (9). Then, there exists a unique bounded solution $\boldsymbol{P}(\boldsymbol{x}, \boldsymbol{y}, t)$ of (20), and $\boldsymbol{P}(\boldsymbol{x}, \boldsymbol{y}, t)$ is given by

$$
\boldsymbol{P}(\boldsymbol{x}, \boldsymbol{y}, t)=\int_{D^{2}} \boldsymbol{G}\left(\boldsymbol{x}, t ; \boldsymbol{x}^{\prime}, t_{0}\right)\left[\boldsymbol{P}_{0}^{+}\left(\boldsymbol{x}^{\prime}, \boldsymbol{y}^{\prime}\right)+\boldsymbol{M}\left(\boldsymbol{x}^{\prime}, \boldsymbol{y}^{\prime}, t\right)\right]^{+} \boldsymbol{G}^{T}\left(\boldsymbol{y}, t ; \boldsymbol{y}^{\prime}, t_{0}\right) d \boldsymbol{x}^{\prime} d \boldsymbol{y}^{\prime}
$$


where

$$
\begin{aligned}
\boldsymbol{M}(\boldsymbol{x}, \boldsymbol{y}, t) & =\int_{t_{0}}^{t} \int_{D^{2}} \boldsymbol{G}^{T}\left(\boldsymbol{\eta}, \tau ; \boldsymbol{x}, t_{0}\right) \overline{\boldsymbol{R}}_{d}(\boldsymbol{\eta}, \boldsymbol{\alpha}, \tau) \boldsymbol{G}\left(\boldsymbol{\alpha}, \tau ; \boldsymbol{y}, t_{0}\right) d \boldsymbol{\eta} d \boldsymbol{\alpha} d \tau \\
& +\int_{t_{0}}^{t} \int_{S^{2}} \boldsymbol{G}^{T}\left(\boldsymbol{\xi}, \tau ; \boldsymbol{x}, t_{0}\right) \overline{\boldsymbol{R}}_{b}(\boldsymbol{\xi}, \boldsymbol{\gamma}, \tau) \boldsymbol{G}\left(\boldsymbol{\gamma}, \tau ; \boldsymbol{y}, t_{0}\right) d S_{\boldsymbol{\eta}} d S_{\boldsymbol{\gamma}} d \tau
\end{aligned}
$$

and the Green function $\boldsymbol{G}\left(\boldsymbol{x}, t ; \boldsymbol{x}^{\prime}, t^{\prime}\right)$ satisfies

$$
\begin{aligned}
& \frac{\partial \boldsymbol{G}\left(\boldsymbol{x}, t ; \boldsymbol{x}^{\prime}, t^{\prime}\right)}{\partial \bar{t}}=\overline{\boldsymbol{A}}_{\boldsymbol{x}} \boldsymbol{G}\left(\boldsymbol{x}, t ; \boldsymbol{x}^{\prime}, t^{\prime}\right), \\
& \boldsymbol{G}\left(\boldsymbol{x}, t^{\prime} ; \boldsymbol{x}^{\prime}, t^{\prime}\right)=\boldsymbol{I} \delta\left(\boldsymbol{x}-\boldsymbol{x}^{\prime}\right), \\
& \overline{\boldsymbol{\beta}}_{\boldsymbol{\xi}} \boldsymbol{G}\left(\boldsymbol{\xi}, t ; \boldsymbol{x}^{\prime}, t^{\prime}\right)=0 .
\end{aligned}
$$

Proof. See Appendix D.

\section{Optimal Observer Design}

\subsection{Distributed measurements}

We reformulate the filter design problem as an optimal control to avoid statistical assumptions on the disturbance vectors $\boldsymbol{w}_{d}(\boldsymbol{x}, t), \boldsymbol{w}_{0}(\boldsymbol{x}), \boldsymbol{w}_{b}(\boldsymbol{\xi}, t), \boldsymbol{\varepsilon}_{d}(\boldsymbol{x}, t)$, and $\varepsilon_{b}(\boldsymbol{\xi}, t)$, and to avoid a probabilistic treatment. As such, we desire to minimize the following cost functional $J$ defined in (10) with respect to $\boldsymbol{\chi}(\boldsymbol{x}, t), \boldsymbol{u}_{d}(\boldsymbol{x}, t)$, and $\boldsymbol{u}_{b}(\boldsymbol{\xi}, t)$ where we recast the functional $L$ to

$$
\begin{aligned}
L & =\frac{1}{2} \int_{D^{2}}\left\langle\boldsymbol{u}_{d}(\boldsymbol{x}, t), \boldsymbol{R}_{d}^{+}(\boldsymbol{x}, \boldsymbol{y}, t) \boldsymbol{u}_{d}(\boldsymbol{y}, t)\right\rangle d \boldsymbol{x} d \boldsymbol{y}+\frac{1}{2} \int_{S^{2}}\left\langle\boldsymbol{u}_{b}(\boldsymbol{\xi}, t), \boldsymbol{R}_{b}^{+}(\boldsymbol{\xi}, \boldsymbol{\alpha}, t) \boldsymbol{u}_{b}(\boldsymbol{\alpha}, t)\right\rangle d S_{\boldsymbol{\xi}} d S_{\boldsymbol{\alpha}} \\
& +\frac{1}{2} \int_{D^{2}}\left\langle\left[\boldsymbol{z}_{d}(\boldsymbol{x}, t)-\boldsymbol{H}_{d}(\boldsymbol{x}, t) \boldsymbol{\chi}(\boldsymbol{x}, t)\right], \boldsymbol{Q}_{d}^{+}(\boldsymbol{x}, \boldsymbol{y}, t)\left[\boldsymbol{z}_{d}(\boldsymbol{y}, t)-\boldsymbol{H}_{d}(\boldsymbol{y}, t) \boldsymbol{\chi}(\boldsymbol{y}, t)\right]\right\rangle d \boldsymbol{x} d \boldsymbol{y} \\
& +\frac{1}{2} \int_{S^{2}}\left\langle\left[\boldsymbol{z}_{b}(\boldsymbol{\xi}, t)-\boldsymbol{H}_{b}(\boldsymbol{\xi}, t) \boldsymbol{\chi}(\boldsymbol{\xi}, t)\right], \boldsymbol{Q}_{b}^{+}(\boldsymbol{\xi}, \boldsymbol{\alpha}, t)\left[\boldsymbol{z}_{b}(\boldsymbol{\alpha}, t)-\boldsymbol{H}_{b}(\boldsymbol{\alpha}, t) \boldsymbol{\chi}(\boldsymbol{\alpha}, t)\right]\right\rangle d S_{\boldsymbol{\xi}} d S_{\boldsymbol{\alpha}}
\end{aligned}
$$

subject to the constraints

$$
\begin{aligned}
& \frac{\partial \boldsymbol{\chi}(\boldsymbol{x}, t)}{\partial t}=\boldsymbol{A}_{\boldsymbol{x}} \boldsymbol{\chi}(\boldsymbol{x}, t)+\boldsymbol{u}_{d}(\boldsymbol{x}, t), \quad \forall \boldsymbol{x} \in D, \\
& \boldsymbol{\chi}\left(\boldsymbol{x}, t_{0}\right)=\boldsymbol{\chi}_{0}(\boldsymbol{x})+\boldsymbol{w}_{0}(\boldsymbol{x}), \quad \forall \boldsymbol{x} \in D, \\
& \boldsymbol{\beta}_{\boldsymbol{\xi}} \boldsymbol{\chi}(\boldsymbol{\xi}, t)=\boldsymbol{u}_{b}(\boldsymbol{\xi}, t), \quad \forall \boldsymbol{\xi} \in S .
\end{aligned}
$$

To remove the above constraints, we introduce the Lagrange multiplier $\boldsymbol{\lambda}(\boldsymbol{w}, t)$ with $\boldsymbol{w} \in D$ or $\boldsymbol{w} \in S$ and construct the following extended functional

$$
\begin{aligned}
L_{1}= & L+\int_{D}\left\langle\boldsymbol{\lambda}(\boldsymbol{x}, t), \frac{\partial \boldsymbol{\chi}(\boldsymbol{x}, t)}{\partial t}-\boldsymbol{A}_{\boldsymbol{x}} \boldsymbol{\chi}(\boldsymbol{x}, t)-\boldsymbol{u}_{d}(\boldsymbol{x}, t)\right\rangle d \boldsymbol{x} \\
& +\int_{S}\left\langle\boldsymbol{\lambda}(\boldsymbol{\xi}, t),-\boldsymbol{\beta}_{\boldsymbol{\xi}} \boldsymbol{\chi}(\boldsymbol{\xi}, t)+\boldsymbol{u}_{b}(\boldsymbol{\xi}, t)\right\rangle d S_{\boldsymbol{\xi}} .
\end{aligned}
$$


Thus, the problem of minimizing the cost functional (10) with $L$ given in (24) subject to the constraints (25) is equivalent to the one of minimizing the cost functional

$$
J_{1}=\int_{t_{0}}^{t_{f}} L_{1} d t+J_{0}
$$

without any constraints. Deriving and setting the weak variation $\delta J_{1}$, see Appendix E, with respect to $\boldsymbol{\chi}(\boldsymbol{x}, t), \boldsymbol{\lambda}(\boldsymbol{x}, t), \boldsymbol{u}_{d}(\boldsymbol{x}, t)$, and $\boldsymbol{u}_{b}(\boldsymbol{\xi}, t)$ to zero result in the following necessary conditions referred to as the set of Euler-Lagrange equations for the cost functional $J_{1}$ to be minimized:

$$
\begin{aligned}
& \frac{\partial \boldsymbol{\chi}(\boldsymbol{x}, t)}{\partial t}=\boldsymbol{A}_{\boldsymbol{x}} \boldsymbol{\chi}(\boldsymbol{x}, t)-\int_{D} \boldsymbol{R}_{d}(\boldsymbol{x}, \boldsymbol{y}, t) \boldsymbol{\lambda}(\boldsymbol{y}, t) d \boldsymbol{y} \\
& \boldsymbol{\beta}_{\boldsymbol{\xi}} \boldsymbol{\chi}(\boldsymbol{\xi}, t)=-\int_{S} \boldsymbol{R}_{b}(\boldsymbol{\xi}, \boldsymbol{\alpha}, t) \boldsymbol{\lambda}(\boldsymbol{\alpha}, t) d S_{\boldsymbol{\alpha}}, \quad \forall \boldsymbol{\xi} \in S, \\
& \frac{\partial \boldsymbol{\lambda}(\boldsymbol{x}, t)}{\partial t}=-\boldsymbol{A}_{\boldsymbol{x}}^{*} \boldsymbol{\lambda}(\boldsymbol{x}, t)+\int_{D} \boldsymbol{H}_{d}^{T}(\boldsymbol{x}, t) \boldsymbol{Q}_{d}^{+}(\boldsymbol{x}, \boldsymbol{y}, t)\left[\boldsymbol{z}_{d}(\boldsymbol{y}, t)-\boldsymbol{H}_{d}(\boldsymbol{y}, t) \boldsymbol{\chi}(\boldsymbol{y}, t)\right] d \boldsymbol{y}, \\
& \boldsymbol{\beta}_{\boldsymbol{\xi}}^{*} \boldsymbol{\lambda}(\boldsymbol{\xi}, t)=-\int_{S} \boldsymbol{H}_{b}^{T}(\boldsymbol{\xi}, t) \boldsymbol{Q}_{b}^{+}(\boldsymbol{\xi}, \boldsymbol{\alpha}, t)\left[\boldsymbol{z}_{b}(\boldsymbol{\alpha}, t)-\boldsymbol{H}_{b}(\boldsymbol{\alpha}, t) \boldsymbol{\chi}(\boldsymbol{\alpha}, t)\right] d S_{\boldsymbol{\alpha}}, \\
& \boldsymbol{\lambda}\left(\boldsymbol{x}, t_{0}\right)=-\int_{D} \boldsymbol{P}_{0}^{+}(\boldsymbol{x}, \boldsymbol{y})\left[\boldsymbol{\chi}\left(\boldsymbol{y}, t_{0}\right)-\boldsymbol{\chi}_{0}(\boldsymbol{y})\right] d \boldsymbol{y}, \\
& \boldsymbol{\lambda}\left(\boldsymbol{x}, t_{f}\right)=0 .
\end{aligned}
$$

The whole system (28) is coupled and is not of two initial-value problems but constitutes a single two-point boundary value (TPBV) problem in the time interval $T$. Thus, we will convert the TPBV problem to an initial value problem. We define

$$
\begin{aligned}
& \tilde{\chi}(\boldsymbol{x}, t)=\chi(\boldsymbol{x}, t)-\hat{\chi}(\boldsymbol{x}, t), \\
& \tilde{\chi}(\boldsymbol{\xi}, t)=\chi(\boldsymbol{\xi}, t)-\hat{\chi}(\boldsymbol{\xi}, t),
\end{aligned}
$$

where $\boldsymbol{\chi}(\boldsymbol{x}, t)$ and $\boldsymbol{\chi}(\boldsymbol{\xi}, t)$ are the state vectors of the system (28) (not of the original system (1)). Substituting (29) into (28) results in

$$
\begin{aligned}
& \frac{\partial \tilde{\chi}(\boldsymbol{x}, t)}{\partial t}=-\frac{\partial \hat{\chi}(\boldsymbol{x}, t)}{\partial t}+\boldsymbol{A}_{\boldsymbol{x}} \hat{\chi}(\boldsymbol{x}, t)+\boldsymbol{A}_{\boldsymbol{x}} \tilde{\chi}(\boldsymbol{x}, t)-\int_{D} \boldsymbol{R}_{d}(\boldsymbol{x}, \boldsymbol{y}, t) \boldsymbol{\lambda}(\boldsymbol{y}, t) d \boldsymbol{y}, \\
& \boldsymbol{\beta}_{\boldsymbol{\xi}} \tilde{\chi}(\boldsymbol{\xi}, t)=-\boldsymbol{\beta}_{\boldsymbol{\xi}} \hat{\chi}(\boldsymbol{x}, t)-\int_{S} \boldsymbol{R}_{b}(\boldsymbol{\xi}, \boldsymbol{\alpha}, t) \boldsymbol{\lambda}(\boldsymbol{\alpha}, t) d S_{\boldsymbol{\alpha}}, \quad \forall \boldsymbol{\xi} \in S,
\end{aligned}
$$

and

$$
\begin{aligned}
\frac{\partial \boldsymbol{\lambda}(\boldsymbol{x}, t)}{\partial t}= & -\boldsymbol{A}_{\boldsymbol{x}}^{*} \boldsymbol{\lambda}(\boldsymbol{x}, t)+\int_{D} \boldsymbol{H}_{d}^{T}(\boldsymbol{x}, t) \boldsymbol{Q}_{d}^{+}(\boldsymbol{x}, \boldsymbol{y}, t)\left[\boldsymbol{z}_{d}(\boldsymbol{y}, t)-\boldsymbol{H}_{d}(\boldsymbol{y}, t) \hat{\boldsymbol{\chi}}(\boldsymbol{y}, t)\right] d \boldsymbol{y} \\
& -\int_{D} \overline{\boldsymbol{Q}}_{d}(\boldsymbol{x}, \boldsymbol{y}, t) \tilde{\boldsymbol{\chi}}(\boldsymbol{y}, t) d \boldsymbol{y}, \\
\boldsymbol{\beta}_{\boldsymbol{\xi}}^{*} \boldsymbol{\lambda}(\boldsymbol{\xi}, t)= & -\int_{S} \boldsymbol{H}_{b}^{T}(\boldsymbol{\xi}, t) \boldsymbol{Q}_{b}^{+}(\boldsymbol{\xi}, \boldsymbol{\alpha}, t)\left[\boldsymbol{z}_{b}(\boldsymbol{\alpha}, t)-\boldsymbol{H}_{b}(\boldsymbol{\alpha}, t) \hat{\boldsymbol{\chi}}(\boldsymbol{\alpha}, t)\right] d S_{\boldsymbol{\alpha}} \\
& +\int_{S} \overline{\boldsymbol{Q}}_{b}(\boldsymbol{\xi}, \boldsymbol{\alpha}, t) \tilde{\boldsymbol{\chi}}(\boldsymbol{\alpha}, t) d S_{\boldsymbol{\alpha}},
\end{aligned}
$$




$$
\begin{aligned}
& \boldsymbol{\lambda}\left(\boldsymbol{x}, t_{0}\right)=-\int_{D} \boldsymbol{P}_{0}^{+}(\boldsymbol{x}, \boldsymbol{y})\left[\hat{\boldsymbol{\chi}}\left(\boldsymbol{y}, t_{0}\right)-\boldsymbol{\chi}_{0}(\boldsymbol{y})\right] d \boldsymbol{y}-\int_{D} \boldsymbol{P}_{0}^{+}(\boldsymbol{x}, \boldsymbol{y}) \tilde{\chi}\left(\boldsymbol{y}, t_{0}\right) d \boldsymbol{y}, \\
& \boldsymbol{\lambda}\left(\boldsymbol{x}, t_{f}\right)=0
\end{aligned}
$$

where $\overline{\boldsymbol{Q}}_{d}(\boldsymbol{x}, \boldsymbol{y}, t)$ and $\overline{\boldsymbol{Q}}_{b}(\boldsymbol{\xi}, \boldsymbol{\alpha}, t)$ are defined in (5). The set of equations (30) suggests that we choose

$$
\begin{aligned}
& \frac{\partial \hat{\boldsymbol{\chi}}(\boldsymbol{x}, t)}{\partial t}=\boldsymbol{A}_{\boldsymbol{x}} \hat{\chi}(\boldsymbol{x}, t)+\boldsymbol{\Omega}(\boldsymbol{x}, t), \\
& \boldsymbol{\beta}_{\boldsymbol{\xi}} \hat{\boldsymbol{\chi}}(\boldsymbol{\xi}, t)=0, \quad \forall \boldsymbol{\xi} \in S, \\
& \hat{\boldsymbol{\chi}}\left(\boldsymbol{x}, t_{0}\right)=\boldsymbol{\chi}_{0}(\boldsymbol{x}),
\end{aligned}
$$

where $\boldsymbol{\Omega}(\boldsymbol{x}, t)$ is a vector function to be determined later. The reason of introducing $\boldsymbol{\Omega}(\boldsymbol{x}, t)$ is that $\boldsymbol{\chi}(\boldsymbol{x}, t)$ and $\boldsymbol{\lambda}(\boldsymbol{x}, t)$ are not independent so as $\tilde{\boldsymbol{\chi}}(\boldsymbol{x}, t)$ and $\boldsymbol{\lambda}(\boldsymbol{x}, t)$. As such, substituting (32) into (30) results in

$$
\begin{aligned}
& \frac{\partial \tilde{\chi}(\boldsymbol{x}, t)}{\partial t}=\boldsymbol{A}_{\boldsymbol{x}} \tilde{\chi}(\boldsymbol{x}, t)-\int_{D} \boldsymbol{R}_{d}(\boldsymbol{x}, \boldsymbol{y}, t) \boldsymbol{\lambda}(\boldsymbol{y}, t) d \boldsymbol{y}-\boldsymbol{\Omega}(\boldsymbol{x}, t), \\
& \boldsymbol{\beta}_{\boldsymbol{\xi}} \tilde{\chi}(\boldsymbol{\xi}, t)=-\int_{S} \boldsymbol{R}_{b}(\boldsymbol{\xi}, \boldsymbol{\alpha}, t) \boldsymbol{\lambda}(\boldsymbol{\alpha}, t) d S_{\boldsymbol{\alpha}}, \quad \forall \boldsymbol{\xi} \in S .
\end{aligned}
$$

We now convert (33) and (31) to an initial value problem. As such, the third equation of (31) suggests that we introduce the following coordinate transformation

$$
\tilde{\chi}(\boldsymbol{x}, t)=-\int_{D} \boldsymbol{P}(\boldsymbol{x}, \boldsymbol{y}, t) \boldsymbol{\lambda}(\boldsymbol{y}, t) d \boldsymbol{y}
$$

where $\boldsymbol{P}(\boldsymbol{x}, \boldsymbol{y}, t)$ is symmetric and positive semidefinite to be determined so that $\boldsymbol{\lambda}(\boldsymbol{x}, t)$ satisfies the last four equations of (33).

\subsubsection{Determination of $\boldsymbol{P}\left(\boldsymbol{x}, \boldsymbol{y}, t_{0}\right)$}

The fifth equation of (33) and (34) suggest that we choose the initial condition for $\boldsymbol{P}(\boldsymbol{x}, \boldsymbol{y}, t)$ as:

$$
\boldsymbol{P}\left(\boldsymbol{x}, \boldsymbol{y}, t_{0}\right)=\boldsymbol{P}_{0}(\boldsymbol{x}, \boldsymbol{y}) .
$$

It is seen from (35) that $\boldsymbol{\lambda}(\boldsymbol{x}, \boldsymbol{y}, t)$ satisfies the initial condition specified by the fifth equation of (33). At $t=t_{f}$, substituting $\boldsymbol{\lambda}\left(\boldsymbol{x}, t_{f}\right)=0$, see the last equation of (33), to (34) results in $\tilde{\chi}\left(\boldsymbol{x}, t_{f}\right)=0$. This implies that $\hat{\chi}\left(\boldsymbol{x}, t_{f}\right)=\boldsymbol{\chi}\left(\boldsymbol{x}, t_{f}\right)$, i.e., $\hat{\chi}(\boldsymbol{x}, t)$ is the exact estimate of $\chi(\boldsymbol{x}, t)$ at $t=t_{f}$.

\subsubsection{Determination of $\boldsymbol{\beta}_{\xi} P(\xi, y, t)$}

We need to determine the condition for $\boldsymbol{\beta}_{\boldsymbol{\xi}} \boldsymbol{P}(\boldsymbol{\xi}, \boldsymbol{y}, t)$ so that the second equation of (33) satisfies. As such, substituting $\boldsymbol{x}$ by $\boldsymbol{\xi}$ into (34) then applying the operator $\boldsymbol{\beta}_{\boldsymbol{\xi}}$ to the resulting equation yields

$$
\boldsymbol{\beta}_{\xi} \tilde{\chi}(\boldsymbol{\xi}, t)=-\int_{D} \boldsymbol{\beta}_{\boldsymbol{\xi}} \boldsymbol{P}(\boldsymbol{\xi}, \boldsymbol{y}, t) \boldsymbol{\lambda}(\boldsymbol{y}, t) d \boldsymbol{y} .
$$


Next, applying the formula (7) to the term $\int_{S} \boldsymbol{R}_{b}(\boldsymbol{\xi}, \boldsymbol{\alpha}, t) \boldsymbol{\lambda}(\boldsymbol{\alpha}, t) d S_{\boldsymbol{\alpha}}$ in the second equation of (33) yields

$$
\int_{D} \boldsymbol{\sigma}(\boldsymbol{\psi}(\boldsymbol{y})) \boldsymbol{R}_{b}(\boldsymbol{\xi}, \boldsymbol{y}, t) \boldsymbol{\lambda}(\boldsymbol{y}, t) d \boldsymbol{y}=\int_{S} \boldsymbol{R}_{b}(\boldsymbol{\xi}, \boldsymbol{\alpha}, t) \boldsymbol{\lambda}(\boldsymbol{\alpha}, t) d S_{\boldsymbol{\alpha}}
$$

Now substituting (37) into the second equation of (33) gives

$$
\boldsymbol{\beta}_{\xi} \tilde{\chi}(\boldsymbol{\xi}, t)=-\int_{D} \boldsymbol{\sigma}(\boldsymbol{\psi}(\boldsymbol{y})) \boldsymbol{R}_{b}(\boldsymbol{\xi}, \boldsymbol{y}, t) \boldsymbol{\lambda}(\boldsymbol{y}, t) d \boldsymbol{y}
$$

Comparing (38) with (36) suggests that we choose the condition for $\boldsymbol{\beta}_{\boldsymbol{\xi}} \boldsymbol{P}(\boldsymbol{\xi}, \boldsymbol{y}, t)$ as

$$
\boldsymbol{\beta}_{\boldsymbol{\xi}} \boldsymbol{P}(\boldsymbol{\xi}, \boldsymbol{y}, t)=\boldsymbol{\sigma}(\boldsymbol{\psi}(\boldsymbol{y})) \boldsymbol{R}_{b}(\boldsymbol{\xi}, \boldsymbol{y}, t) .
$$

\subsubsection{Determination of $\boldsymbol{P}(\boldsymbol{x}, \boldsymbol{y}, t)$}

We now determine $\boldsymbol{P}(\boldsymbol{x}, \boldsymbol{y}, t)$ so that $\boldsymbol{\lambda}(\boldsymbol{x}, t)$ satisfies (33) and (31). Differentiating both sides of (34) along the solutions of (33) and (31) gives

$$
\begin{aligned}
& \frac{\partial \tilde{\boldsymbol{\chi}}(\boldsymbol{x}, t)}{\partial t}=-\int_{D} \frac{\partial \boldsymbol{P}(\boldsymbol{x}, \boldsymbol{y}, t)}{\partial t} \boldsymbol{\lambda}(\boldsymbol{y}, t) d \boldsymbol{y}+\int_{D} \boldsymbol{P}(\boldsymbol{x}, \boldsymbol{y}, t) \boldsymbol{A}_{\boldsymbol{y}}^{*} \boldsymbol{\lambda}(\boldsymbol{y}, t) d \boldsymbol{y} \\
& \quad-\int_{D^{2}} \boldsymbol{P}(\boldsymbol{x}, \boldsymbol{y}, t) \boldsymbol{H}_{d}^{T}(\boldsymbol{y}, t) \boldsymbol{Q}_{d}^{+}\left(\boldsymbol{y}, \boldsymbol{y}^{\prime}, t\right)\left[\boldsymbol{z}_{d}\left(\boldsymbol{y}^{\prime}, t\right)-\boldsymbol{H}_{d}\left(\boldsymbol{y}^{\prime}, t\right) \hat{\boldsymbol{\chi}}\left(\boldsymbol{y}^{\prime}, t\right)\right] d \boldsymbol{y}^{\prime} d \boldsymbol{y} \\
& \quad-\int_{D^{3}} \boldsymbol{P}\left(\boldsymbol{x}, \boldsymbol{y}^{\prime}, t\right) \overline{\boldsymbol{Q}}_{d}\left(\boldsymbol{y}^{\prime}, \boldsymbol{y}^{\prime \prime}, t\right) \boldsymbol{P}\left(\boldsymbol{y}^{\prime \prime}, \boldsymbol{y}, t\right) \boldsymbol{\lambda}(\boldsymbol{y}, t) d \boldsymbol{y}^{\prime \prime} d \boldsymbol{y}^{\prime} d \boldsymbol{y},
\end{aligned}
$$

where we have used (34). Taking inner product both sides of (40) with $\boldsymbol{\lambda}(\boldsymbol{x}, t)$ then integrating over $D$ yields

$$
\begin{aligned}
& \int_{D}\left\langle\boldsymbol{\lambda}(\boldsymbol{x}, t), \frac{\partial \tilde{\boldsymbol{\chi}}(\boldsymbol{x}, t)}{\partial t}\right\rangle d \boldsymbol{x}=-\int_{D^{2}}\left\langle\boldsymbol{\lambda}(\boldsymbol{x}, t), \frac{\partial \boldsymbol{P}(\boldsymbol{x}, \boldsymbol{y}, t)}{\partial t} \boldsymbol{\lambda}(\boldsymbol{y}, t)\right\rangle d \boldsymbol{x} d \boldsymbol{y} \\
& +\int_{D^{2}}\left\langle\boldsymbol{\lambda}(\boldsymbol{x}, t), \boldsymbol{P}(\boldsymbol{x}, \boldsymbol{y}, t) \boldsymbol{A}_{\boldsymbol{y}}^{*} \boldsymbol{\lambda}(\boldsymbol{y}, t)\right\rangle d \boldsymbol{x} d \boldsymbol{y} \\
& -\int_{D^{2}}\left\langle\boldsymbol{\lambda}(\boldsymbol{x}, t), \int_{D} \boldsymbol{P}(\boldsymbol{x}, \boldsymbol{y}, t) \boldsymbol{H}_{d}^{T}(\boldsymbol{y}, t) \boldsymbol{Q}_{d}^{+}\left(\boldsymbol{y}, \boldsymbol{y}^{\prime}, t\right)\left[\boldsymbol{z}_{d}\left(\boldsymbol{y}^{\prime}, t\right)-\boldsymbol{H}_{d}\left(\boldsymbol{y}^{\prime}, t\right) \hat{\boldsymbol{\chi}}\left(\boldsymbol{y}^{\prime}, t\right)\right] d \boldsymbol{y}^{\prime}\right\rangle d \boldsymbol{x} d \boldsymbol{y} \\
& -\int_{D^{2}}\left\langle\boldsymbol{\lambda}(\boldsymbol{x}, t), \int_{D^{2}} \boldsymbol{P}\left(\boldsymbol{x}, \boldsymbol{y}^{\prime}, t\right) \overline{\boldsymbol{Q}}_{d}\left(\boldsymbol{y}^{\prime}, \boldsymbol{y}^{\prime \prime}, t\right) \boldsymbol{P}\left(\boldsymbol{y}^{\prime \prime}, \boldsymbol{y}, t\right) \boldsymbol{\lambda}(\boldsymbol{y}, t) d \boldsymbol{y}^{\prime \prime} d \boldsymbol{y}^{\prime}\right\rangle d \boldsymbol{x} d \boldsymbol{y} .
\end{aligned}
$$

Applying Lemma 3.1 to the second integral term in the right hand side of (41) gives

$$
\begin{aligned}
\int_{D^{2}}\left\langle\boldsymbol{\lambda}(\boldsymbol{x}, t), \boldsymbol{P}(\boldsymbol{x}, \boldsymbol{y}, t) \boldsymbol{A}_{\boldsymbol{y}}^{*} \boldsymbol{\lambda}(\boldsymbol{y}, t)\right\rangle d \boldsymbol{x} d \boldsymbol{y}=\int_{D^{2}}\left\langle\boldsymbol{\lambda}(\boldsymbol{x}, t),\left[\boldsymbol{P}(\boldsymbol{x}, \boldsymbol{y}, t) \boldsymbol{A}_{\boldsymbol{y}}^{T}\right] \boldsymbol{\lambda}(\boldsymbol{y}, t)\right\rangle d \boldsymbol{y} d \boldsymbol{x} \\
-\int_{D} \int_{S}\left\langle\boldsymbol{\lambda}(\boldsymbol{x}, t),\left[\boldsymbol{\beta}_{\boldsymbol{\xi}} \boldsymbol{P}(\boldsymbol{\xi}, \boldsymbol{x}, t)\right]^{T} \boldsymbol{\lambda}(\boldsymbol{\xi}, t)\right\rangle d \boldsymbol{x} d S_{\boldsymbol{\xi}} \\
+\int_{D} \int_{S}\left\langle\boldsymbol{\lambda}(\boldsymbol{x}, t), \boldsymbol{P}(\boldsymbol{x}, \boldsymbol{\xi}, t) \boldsymbol{\beta}_{\boldsymbol{\xi}}^{*} \boldsymbol{\lambda}(\boldsymbol{x}, t)\right\rangle d \boldsymbol{x} d S_{\boldsymbol{\xi}} .
\end{aligned}
$$


Substituting the third equation of (33) and (39) into (42) gives

$$
\begin{aligned}
\int_{D^{2}}\langle\boldsymbol{\lambda} & \left.(\boldsymbol{x}, t), \boldsymbol{P}(\boldsymbol{x}, \boldsymbol{y}, t) \boldsymbol{A}_{\boldsymbol{y}}^{*} \boldsymbol{\lambda}(\boldsymbol{y}, t)\right\rangle d \boldsymbol{x} d \boldsymbol{y}=\int_{D^{2}}\left\langle\boldsymbol{\lambda}(\boldsymbol{x}, t),\left[\boldsymbol{P}(\boldsymbol{x}, \boldsymbol{y}, t) \boldsymbol{A}_{\boldsymbol{y}}^{T}\right] \boldsymbol{\lambda}(\boldsymbol{y}, t)\right\rangle d \boldsymbol{y} d \boldsymbol{x} \\
& -\int_{D}\left\langle\boldsymbol{\lambda}(\boldsymbol{x}, t), \int_{S}\left[\boldsymbol{\sigma}(\boldsymbol{\psi}(\boldsymbol{x})) \boldsymbol{R}_{b}(\boldsymbol{\xi}, \boldsymbol{x}, t)\right]^{T} \boldsymbol{\lambda}(\boldsymbol{\xi}, t) d S_{\boldsymbol{\xi}}\right\rangle d \boldsymbol{x} \\
& -\int_{D}\left\langle\boldsymbol{\lambda}(\boldsymbol{x}, t), \int_{S^{2}} \boldsymbol{P}(\boldsymbol{x}, \boldsymbol{\xi}, t) \boldsymbol{H}_{b}^{T}(\boldsymbol{\xi}, t) \boldsymbol{Q}_{b}^{+}(\boldsymbol{\xi}, \boldsymbol{\alpha}, t)\left[\boldsymbol{z}_{b}(\boldsymbol{\alpha}, t)\right.\right. \\
& \left.\left.-\boldsymbol{H}_{b}(\boldsymbol{\alpha}, t) \hat{\boldsymbol{\chi}}(\boldsymbol{\alpha}, t)\right] d S_{\boldsymbol{\alpha}} d S_{\boldsymbol{\xi}}\right\rangle d \boldsymbol{x} \\
& -\int_{D^{2}}\left\langle\boldsymbol{\lambda}(\boldsymbol{x}, t), \int_{S^{2}} \boldsymbol{P}(\boldsymbol{x}, \boldsymbol{\xi}, t) \overline{\boldsymbol{Q}}_{b}(\boldsymbol{\xi}, \boldsymbol{\alpha}, t) \boldsymbol{P}(\boldsymbol{\alpha}, \boldsymbol{y}, t) \boldsymbol{\lambda}(\boldsymbol{y}, t) d S_{\boldsymbol{\alpha}} d S_{\boldsymbol{\xi}}\right\rangle d \boldsymbol{x} d \boldsymbol{y} .
\end{aligned}
$$

Applying the formula (7) to the second term in the right hand side of (43) yields

$$
\begin{aligned}
& \int_{D^{2}}\left\langle\boldsymbol{\lambda}(\boldsymbol{x}, t), \boldsymbol{P}(\boldsymbol{x}, \boldsymbol{y}, t) \boldsymbol{A}_{\boldsymbol{y}}^{*} \boldsymbol{\lambda}(\boldsymbol{y}, t)\right\rangle d \boldsymbol{x} d \boldsymbol{y}=\int_{D^{2}}\left\langle\boldsymbol{\lambda}(\boldsymbol{x}, t),\left[\boldsymbol{P}(\boldsymbol{x}, \boldsymbol{y}, t) \boldsymbol{A}_{\boldsymbol{y}}^{T}\right] \boldsymbol{\lambda}(\boldsymbol{y}, t)\right\rangle d \boldsymbol{y} d \boldsymbol{x} \\
& -\int_{D^{2}}\left\langle\boldsymbol{\lambda}(\boldsymbol{x}, t), \boldsymbol{\sigma}(\boldsymbol{\psi}(\boldsymbol{y})) \boldsymbol{R}_{b}(\boldsymbol{x}, \boldsymbol{y}, t) \boldsymbol{\sigma}(\boldsymbol{\psi}(\boldsymbol{x})) \boldsymbol{\lambda}(\boldsymbol{y}, t)\right\rangle d \boldsymbol{x} d \boldsymbol{y} \\
& -\int_{D}\left\langle\boldsymbol{\lambda}(\boldsymbol{x}, t), \int_{S^{2}} \boldsymbol{P}(\boldsymbol{x}, \boldsymbol{\xi}, t) \boldsymbol{H}_{b}^{T}(\boldsymbol{\xi}, t) \boldsymbol{Q}_{b}^{+}(\boldsymbol{\xi}, \boldsymbol{\alpha}, t)\left[\boldsymbol{z}_{b}(\boldsymbol{\alpha}, t)-\boldsymbol{H}_{b}(\boldsymbol{\alpha}, t) \hat{\boldsymbol{\chi}}(\boldsymbol{\alpha}, t)\right] d S_{\boldsymbol{\alpha}} d S_{\boldsymbol{\xi}}\right\rangle d \boldsymbol{x} \\
& -\int_{D^{2}}\left\langle\boldsymbol{\lambda}(\boldsymbol{x}, t), \int_{S^{2}} \boldsymbol{P}(\boldsymbol{x}, \boldsymbol{\xi}, t) \overline{\boldsymbol{Q}}_{b}(\boldsymbol{\xi}, \boldsymbol{\alpha}, t) \boldsymbol{P}(\boldsymbol{\alpha}, \boldsymbol{y}, t) \boldsymbol{\lambda}(\boldsymbol{y}, t) d S_{\boldsymbol{\alpha}} d S_{\boldsymbol{\xi}}\right\rangle d \boldsymbol{x} d \boldsymbol{y},
\end{aligned}
$$

On the other hand, substituting (34) into the right hand side of the first equation of (33) then taking inner product with $\boldsymbol{\lambda}(\boldsymbol{x}, t)$ and integrating over $D$ yields

$$
\begin{aligned}
\int_{D} & \left\langle\boldsymbol{\lambda}(\boldsymbol{x}, t), \frac{\partial \tilde{\boldsymbol{\chi}}(\boldsymbol{x}, t)}{\partial t}\right\rangle d \boldsymbol{x}=-\int_{D^{2}}\left\langle\boldsymbol{\lambda}(\boldsymbol{x}, t), \boldsymbol{A}_{\boldsymbol{x}} \boldsymbol{P}(\boldsymbol{x}, \boldsymbol{y}, t) \boldsymbol{\lambda}(\boldsymbol{y}, t)\right\rangle d \boldsymbol{x} d \boldsymbol{y} \\
& -\int_{D^{2}}\left\langle\boldsymbol{\lambda}(\boldsymbol{x}, t), \boldsymbol{R}_{d}(\boldsymbol{x}, \boldsymbol{y}, t) \boldsymbol{\lambda}(\boldsymbol{y}, t)\right\rangle d \boldsymbol{x} d \boldsymbol{y}-\int_{D}\langle\boldsymbol{\lambda}(\boldsymbol{x}, t), \boldsymbol{\Omega}(\boldsymbol{x}, t)\rangle d \boldsymbol{x}
\end{aligned}
$$

Since $\boldsymbol{\lambda}(\boldsymbol{x}, t)$ is a nontrivial solution of the third equation of (33), comparing (45) with substitution of (44) into (41) suggests that we choose the term $\boldsymbol{\Omega}(\boldsymbol{x}, t)$ as

$$
\begin{aligned}
\boldsymbol{\Omega}(\boldsymbol{x}, t) & =\int_{D^{2}} \boldsymbol{P}(\boldsymbol{x}, \boldsymbol{y}, t) \boldsymbol{H}_{d}^{T}(\boldsymbol{y}, t) \boldsymbol{Q}_{d}^{+}\left(\boldsymbol{y}, \boldsymbol{y}^{\prime}, t\right)\left[\boldsymbol{z}_{d}\left(\boldsymbol{y}^{\prime}, t\right)-\boldsymbol{H}_{d}\left(\boldsymbol{y}^{\prime}, t\right) \hat{\boldsymbol{\chi}}\left(\boldsymbol{y}^{\prime}, t\right)\right] d \boldsymbol{y}^{\prime} d \boldsymbol{y} \\
& +\int_{S^{2}} \boldsymbol{P}(\boldsymbol{x}, \boldsymbol{\xi}, t) \boldsymbol{H}_{b}^{T}(\boldsymbol{\xi}, t) \boldsymbol{Q}_{b}^{+}(\boldsymbol{\xi}, \boldsymbol{\alpha}, t)\left[\boldsymbol{z}_{b}(\boldsymbol{\alpha}, t)-\boldsymbol{H}_{b}(\boldsymbol{\alpha}, t) \hat{\boldsymbol{\chi}}(\boldsymbol{\alpha}, t)\right] d S_{\boldsymbol{\alpha}} d S_{\boldsymbol{\xi}}
\end{aligned}
$$


and the equation for $\boldsymbol{P}(\boldsymbol{x}, \boldsymbol{y}, t)$ as

$$
\begin{aligned}
& \frac{\partial \boldsymbol{P}(\boldsymbol{x}, \boldsymbol{y}, t)}{\partial t}=\boldsymbol{A}_{\boldsymbol{x}} \boldsymbol{P}(\boldsymbol{x}, \boldsymbol{y}, t)+\boldsymbol{P}(\boldsymbol{x}, \boldsymbol{y}, t) \boldsymbol{A}_{\boldsymbol{y}}^{T}+\boldsymbol{R}_{d}(\boldsymbol{x}, \boldsymbol{y}, t)-\boldsymbol{\sigma}(\boldsymbol{\psi}(\boldsymbol{y})) \boldsymbol{R}_{b}(\boldsymbol{x}, \boldsymbol{y}, t) \boldsymbol{\sigma}(\boldsymbol{\psi}(\boldsymbol{x})) \\
& -\int_{D^{2}} \boldsymbol{P}\left(\boldsymbol{x}, \boldsymbol{y}^{\prime}, t\right) \overline{\boldsymbol{Q}}_{d}\left(\boldsymbol{y}^{\prime}, \boldsymbol{y}^{\prime \prime}, t\right) \boldsymbol{P}\left(\boldsymbol{y}^{\prime \prime}, \boldsymbol{y}, t\right) d \boldsymbol{y}^{\prime \prime} d \boldsymbol{y}^{\prime}-\int_{S^{2}} \boldsymbol{P}(\boldsymbol{x}, \boldsymbol{\xi}, t) \overline{\boldsymbol{Q}}_{b}(\boldsymbol{\xi}, \boldsymbol{\alpha}, t) \boldsymbol{P}(\boldsymbol{\alpha}, \boldsymbol{y}, t) d S_{\boldsymbol{\alpha}} d S_{\boldsymbol{\xi}},
\end{aligned}
$$

which is a Riccati nonlinear PDE. Since solving this PDE is extremely difficult, we propose a special choice of the matrices $\boldsymbol{R}_{d}(\boldsymbol{x}, \boldsymbol{y}, t)$ and $\boldsymbol{R}_{b}(\boldsymbol{\xi}, \boldsymbol{y}, t)$ to simplify the Riccati nonlinear PDE (47) to a Bernoulli nonlinear PDE, of which an analytical solution can be found. So, we choose the matrices $\boldsymbol{R}_{d}(\boldsymbol{x}, \boldsymbol{y}, t)$ and $\boldsymbol{R}_{b}(\boldsymbol{\xi}, \boldsymbol{y}, t)$ as

$\boldsymbol{R}_{d}(\boldsymbol{x}, \boldsymbol{y}, t)=\boldsymbol{L}_{d}(\boldsymbol{x}, \boldsymbol{y}, t) \boldsymbol{P}(\boldsymbol{x}, \boldsymbol{y}, t)+\boldsymbol{P}(\boldsymbol{x}, \boldsymbol{y}, t) \boldsymbol{L}_{d}^{T}(\boldsymbol{x}, \boldsymbol{y}, t)+\boldsymbol{\sigma}(\boldsymbol{\psi}(\boldsymbol{y})) \boldsymbol{R}_{b}(\boldsymbol{x}, \boldsymbol{y}, t) \boldsymbol{\sigma}(\boldsymbol{\psi}(\boldsymbol{x}))$, $\boldsymbol{R}_{b}(\boldsymbol{\xi}, \boldsymbol{y}, t)=\boldsymbol{L}_{b}(\boldsymbol{\xi}, t) \boldsymbol{P}(\boldsymbol{\xi}, \boldsymbol{y}, t)$,

where the matrices $\boldsymbol{L}_{d}(\boldsymbol{x}, \boldsymbol{y}, t)$ and $\boldsymbol{L}_{b}(\boldsymbol{\xi}, t)$ are defined in Item 3) of Assumption 2.1.

The observer is given by substituting $\boldsymbol{\Omega}(\boldsymbol{x}, t)$ defined in (46) into (32) as

$$
\begin{aligned}
& \frac{\partial \hat{\boldsymbol{\chi}}(\boldsymbol{x}, t)}{\partial t}=\boldsymbol{A}_{\boldsymbol{x}} \hat{\boldsymbol{\chi}}(\boldsymbol{x}, t) \\
& \quad+\int_{D^{2}} \boldsymbol{P}(\boldsymbol{x}, \boldsymbol{y}, t) \boldsymbol{H}_{d}^{T}(\boldsymbol{y}, t) \boldsymbol{Q}_{d}^{+}\left(\boldsymbol{y}, \boldsymbol{y}^{\prime}, t\right)\left[\boldsymbol{z}_{d}\left(\boldsymbol{y}^{\prime}, t\right)-\boldsymbol{H}_{d}\left(\boldsymbol{y}^{\prime}, t\right) \hat{\boldsymbol{\chi}}\left(\boldsymbol{y}^{\prime}, t\right)\right] d \boldsymbol{y}^{\prime} d \boldsymbol{y} \\
& \quad+\int_{S^{2}} \boldsymbol{P}(\boldsymbol{x}, \boldsymbol{\xi}, t) \boldsymbol{H}_{b}^{T}(\boldsymbol{\xi}, t) \boldsymbol{Q}_{b}^{+}(\boldsymbol{\xi}, \boldsymbol{\alpha}, t)\left[\boldsymbol{z}_{b}(\boldsymbol{\alpha}, t)-\boldsymbol{H}_{b}(\boldsymbol{\alpha}, t) \hat{\boldsymbol{\chi}}(\boldsymbol{\alpha}, t)\right] d S_{\boldsymbol{\alpha}} d S_{\boldsymbol{\xi}}, \\
& \hat{\boldsymbol{\chi}}\left(\boldsymbol{x}, t_{0}\right)=\boldsymbol{\chi}_{0}(\boldsymbol{x}), \\
& \boldsymbol{\beta}_{\boldsymbol{\xi}} \hat{\boldsymbol{\chi}}(\boldsymbol{\xi}, t)=0,
\end{aligned}
$$

and $\boldsymbol{P}(\boldsymbol{x}, \boldsymbol{y}, t)$ generated by substituting (48) into (47) and (39) with the initial condition (35) as

$$
\begin{aligned}
& \frac{\partial \boldsymbol{P}(\boldsymbol{x}, \boldsymbol{y}, t)}{\partial t}=\overline{\boldsymbol{A}}_{\boldsymbol{x}} \boldsymbol{P}(\boldsymbol{x}, \boldsymbol{y}, t)-\int_{D^{2}} \boldsymbol{P}\left(\boldsymbol{x}, \boldsymbol{y}^{\prime}, t\right) \overline{\boldsymbol{Q}}_{d}\left(\boldsymbol{y}^{\prime}, \boldsymbol{y}^{\prime \prime}, t\right) \boldsymbol{P}\left(\boldsymbol{y}^{\prime \prime}, \boldsymbol{y}, t\right) d \boldsymbol{y}^{\prime \prime} d \boldsymbol{y}^{\prime} \\
& \quad+\boldsymbol{P}(\boldsymbol{x}, \boldsymbol{y}, t) \overline{\boldsymbol{A}}_{\boldsymbol{y}}^{T}-\int_{S^{2}} \boldsymbol{P}(\boldsymbol{x}, \boldsymbol{\xi}, t) \overline{\boldsymbol{Q}}_{b}(\boldsymbol{\xi}, \boldsymbol{\alpha}, t) \boldsymbol{P}(\boldsymbol{\alpha}, \boldsymbol{y}, t) d S_{\boldsymbol{\alpha}} d S_{\boldsymbol{\xi}}, \\
& \boldsymbol{P}\left(\boldsymbol{x}, \boldsymbol{y}, t_{0}\right)=\boldsymbol{P}_{0}(\boldsymbol{x}, \boldsymbol{y}), \\
& \overline{\boldsymbol{\beta}}_{\boldsymbol{\xi}} \boldsymbol{P}(\boldsymbol{\xi}, \boldsymbol{y}, t)=0,
\end{aligned}
$$

where $\overline{\boldsymbol{A}}_{\boldsymbol{x}}$ and $\overline{\boldsymbol{\beta}}_{\boldsymbol{\xi}}$ are defined in (9). Note that (49) is an initial value problem.

\subsection{Observer error dynamics and stability analysis}

\subsubsection{Observer error dynamics}

Define the original observer error

$$
\begin{aligned}
& \chi_{e}(\boldsymbol{x}, t)=\chi(\boldsymbol{x}, t)-\hat{\chi}(\boldsymbol{x}, t), \\
& \chi_{e}(\boldsymbol{\xi}, t)=\chi(\boldsymbol{\xi}, t)-\hat{\chi}(\boldsymbol{\xi}, t),
\end{aligned}
$$


where $\boldsymbol{\chi}(\boldsymbol{x}, t)$ and $\boldsymbol{\chi}(\boldsymbol{\xi}, t)$ are now the state vector of the original system (1). It is noted that in (51), $\chi(\boldsymbol{x}, t)$ and $\boldsymbol{\chi}(\boldsymbol{\xi}, t)$ are the state vectors of the original system (1) (not of the system (28)). Differentiating both sides of the first equation of (51) with respect to $t$ along the solutions of the observer system (49), the original system (1), and the measurement system (2) yields

$$
\begin{aligned}
\frac{\partial \boldsymbol{\chi}_{e}(\boldsymbol{x}, t)}{\partial t} & =\boldsymbol{A}_{\boldsymbol{x}} \boldsymbol{\chi}_{e}(\boldsymbol{x}, t)-\int_{D^{2}} \boldsymbol{P}(\boldsymbol{x}, \boldsymbol{y}, t) \overline{\boldsymbol{Q}}_{d}\left(\boldsymbol{y}, \boldsymbol{y}^{\prime}, t\right) \boldsymbol{\chi}_{e}\left(\boldsymbol{y}^{\prime}, t\right) d \boldsymbol{y}^{\prime} d \boldsymbol{y} \\
& -\int_{S^{2}} \boldsymbol{P}(\boldsymbol{x}, \boldsymbol{\xi}, t) \overline{\boldsymbol{Q}}_{b}(\boldsymbol{\xi}, \boldsymbol{\alpha}, t) \boldsymbol{\chi}_{e}(\boldsymbol{\alpha}, t) d S_{\boldsymbol{\alpha}} d S_{\boldsymbol{\xi}}+\boldsymbol{w}_{d}(\boldsymbol{x}, t) \\
& -\int_{D^{2}} \boldsymbol{P}(\boldsymbol{x}, \boldsymbol{y}, t) \boldsymbol{H}_{d}^{T}(\boldsymbol{y}, t) \boldsymbol{Q}_{d}^{+}\left(\boldsymbol{y}, \boldsymbol{y}^{\prime}, t\right) \boldsymbol{\varepsilon}_{d}\left(\boldsymbol{y}^{\prime}, t\right) d \boldsymbol{y}^{\prime} d \boldsymbol{y} \\
& -\int_{S^{2}} \boldsymbol{P}(\boldsymbol{x}, \boldsymbol{\xi}, t) \boldsymbol{H}_{b}^{T}(\boldsymbol{\xi}, t) \boldsymbol{Q}_{b}^{+}(\boldsymbol{\xi}, \boldsymbol{\alpha}, t) \boldsymbol{\varepsilon}_{b}(\boldsymbol{\alpha}, t) d S_{\boldsymbol{\alpha}} d S_{\boldsymbol{\xi}} \\
\boldsymbol{\chi}_{e}\left(\boldsymbol{x}, t_{0}\right)= & \boldsymbol{w}_{0}(\boldsymbol{x}) \\
\boldsymbol{\beta}_{\boldsymbol{\xi}} \chi_{e}(\boldsymbol{\xi}, t) & =\boldsymbol{w}_{b}(\boldsymbol{\xi}, t) .
\end{aligned}
$$

\subsubsection{Lyapunov stability analysis}

To analyze stability of the observer error system (52) at the origin, we consider the following Lyapunov functional candidate

$$
V(t)=\int_{D^{2}}\left\langle\chi_{e}(\boldsymbol{x}, t), \boldsymbol{P}^{+}(\boldsymbol{x}, \boldsymbol{y}, t) \boldsymbol{\chi}_{e}(\boldsymbol{y}, t)\right\rangle d \boldsymbol{x} d \boldsymbol{y}
$$

where $\boldsymbol{P}(\boldsymbol{x}, \boldsymbol{y}, t)$ is the symmetric and positive definite solution of the third equation of (52). A calculation shows that differentiating both sides of (53) with respect time $t$ results in

$$
\begin{aligned}
& \frac{d V(t)}{d t}=-\int_{D^{2}}\left\langle\boldsymbol{\chi}_{e}(\boldsymbol{x}, t),\left(\overline{\boldsymbol{Q}}_{d}(\boldsymbol{x}, \boldsymbol{y}, t)+\overline{\boldsymbol{R}}_{d}(\boldsymbol{x}, \boldsymbol{y}, t)\right) \boldsymbol{\chi}_{e}(\boldsymbol{y}, t)\right\rangle d \boldsymbol{x} d \boldsymbol{y} \\
& -\int_{S^{2}}\left[\left\langle\boldsymbol{\chi}_{e}(\boldsymbol{\xi}, t), \overline{\boldsymbol{Q}}_{b}(\boldsymbol{\xi}, \boldsymbol{\alpha}, t) \boldsymbol{\chi}_{e}(\boldsymbol{\alpha}, t)\right\rangle+2\left\langle\boldsymbol{\varepsilon}_{b}(\boldsymbol{\xi}, t), \boldsymbol{Q}_{b}^{+}(\boldsymbol{\xi}, \boldsymbol{\alpha}, t) \boldsymbol{H}_{b}(\boldsymbol{\alpha}, t) \boldsymbol{\chi}_{e}(\boldsymbol{\alpha}, t)\right\rangle\right] d_{\boldsymbol{\alpha}} d S_{\boldsymbol{\xi}} \\
& -2 \int_{D^{2}}\left[\left\langle\boldsymbol{\varepsilon}_{d}(\boldsymbol{x}, t), \boldsymbol{Q}_{d}^{+}(\boldsymbol{x}, \boldsymbol{y}, t) \boldsymbol{H}_{d}(\boldsymbol{y}, t) \boldsymbol{\chi}_{e}(\boldsymbol{y}, t)\right\rangle-\left\langle\boldsymbol{w}_{d}(\boldsymbol{x}, t), \boldsymbol{P}^{+}(\boldsymbol{x}, \boldsymbol{y}, t) \boldsymbol{\chi}_{e}(\boldsymbol{y}, t)\right\rangle\right] d \boldsymbol{x} d \boldsymbol{y},
\end{aligned}
$$

where

$$
\begin{aligned}
\overline{\boldsymbol{R}}_{d}(\boldsymbol{x}, \boldsymbol{y}, t) & =\int_{D^{2}} \boldsymbol{P}^{+}\left(\boldsymbol{x}, \boldsymbol{x}^{\prime}, t\right)\left(\boldsymbol{L}_{d}\left(\boldsymbol{x}^{\prime}, \boldsymbol{y}^{\prime}, t\right) \boldsymbol{P}\left(\boldsymbol{x}^{\prime}, \boldsymbol{y}^{\prime}, t\right)+\boldsymbol{P}\left(\boldsymbol{x}^{\prime}, \boldsymbol{y}^{\prime}, t\right) \boldsymbol{L}_{d}^{T}\left(\boldsymbol{x}^{\prime}, \boldsymbol{y}^{\prime}, t\right)\right) \\
& \times \boldsymbol{P}^{+}\left(\boldsymbol{y}^{\prime}, \boldsymbol{y}, t\right) d \boldsymbol{x}^{\prime} d \boldsymbol{y}^{\prime}
\end{aligned}
$$

which is symmetric and positive definite. Since $\boldsymbol{\varepsilon}_{d}(\boldsymbol{x}, t) \in L_{2}^{m_{d}}(D \times T), \boldsymbol{w}_{d}(\boldsymbol{x}, t) \in$ $L_{2}^{r}(D \times T)$, and $\boldsymbol{\varepsilon}_{b}(\boldsymbol{x}, t) \in L_{2}^{m_{b}}(S \times T)$, an the matrices $\boldsymbol{P}^{+}(\boldsymbol{x}, \boldsymbol{y}, t), \overline{\boldsymbol{Q}}_{d}(\boldsymbol{x}, \boldsymbol{y}, t)$, 
$\overline{\boldsymbol{Q}}_{b}(\boldsymbol{\xi}, \boldsymbol{\alpha}, t)$, and $\overline{\boldsymbol{R}}_{d}(\boldsymbol{x}, \boldsymbol{y}, t)$ are symmetric and positive definite, we conclude from (53) and (54) that the observer error vector $\chi_{e}(\boldsymbol{x}, t)$ exponentially converges in $L_{2}$ norm to a ball centered the origin. The radius of the ball can be made arbitrarily small by a choice of sufficiently large $\overline{\boldsymbol{Q}}_{d}(\boldsymbol{x}, \boldsymbol{y}, t), \overline{\boldsymbol{Q}}_{b}(\boldsymbol{\xi}, \boldsymbol{\alpha}, t)$, and $\boldsymbol{R}_{d}(\boldsymbol{x}, \boldsymbol{y}, t)$. Moreover, convergence of $\boldsymbol{\chi}_{e}(\boldsymbol{x}, t)$ also implies that of $\boldsymbol{\chi}_{e}(\boldsymbol{\xi}, t)$. This can be seen by applying the formula (7) to (53).

\section{Discrete measurements}

We now address the problem of $M_{d}$ measurements taken at $\boldsymbol{x}_{1}, \boldsymbol{x}_{2}, \ldots, \boldsymbol{x}_{M_{d}}$ discrete locations over $D$ and $M_{b}$ measurements taken at $\boldsymbol{\xi}_{1}, \boldsymbol{\xi}_{2}, \ldots, \boldsymbol{\xi}_{M_{b}}$ discrete locations over $S$. As such, the measurement equation (2) is changed to

$$
\begin{aligned}
& \boldsymbol{z}_{d}\left(\boldsymbol{x}_{i}, t\right)=\boldsymbol{H}_{d}\left(\boldsymbol{x}_{i}, t\right) \boldsymbol{\chi}\left(\boldsymbol{x}_{i}, t\right)+\boldsymbol{\varepsilon}_{d}\left(\boldsymbol{x}_{i}, t\right), i=1, \ldots, M_{d} \\
& \boldsymbol{z}_{b}\left(\boldsymbol{\xi}_{i}, t\right)=\boldsymbol{H}_{b}\left(\boldsymbol{\xi}_{i}, t\right) \boldsymbol{\chi}\left(\boldsymbol{\xi}_{i}, t\right)+\boldsymbol{\varepsilon}_{b}\left(\boldsymbol{\xi}_{i}, t\right), i=1, \ldots, M_{b} .
\end{aligned}
$$

Thus, by setting

$$
\begin{aligned}
& \boldsymbol{z}_{d}(\boldsymbol{x}, t)=\sum_{i=1}^{M_{d}} \boldsymbol{z}\left(\boldsymbol{x}_{i}, t\right) \boldsymbol{I} \delta\left(\boldsymbol{x}-\boldsymbol{x}_{i}\right), \boldsymbol{H}_{d}(\boldsymbol{x}, t) \boldsymbol{\chi}(\boldsymbol{x}, t)=\sum_{i=1}^{M_{d}} \boldsymbol{H}_{d}\left(\boldsymbol{x}_{i}, t\right) \boldsymbol{\chi}\left(\boldsymbol{x}_{i}, t\right) \boldsymbol{I} \delta\left(\boldsymbol{x}-\boldsymbol{x}_{i}\right), \\
& \boldsymbol{z}_{b}(\boldsymbol{\xi}, t)=\sum_{i=1}^{M_{b}} \boldsymbol{z}\left(\boldsymbol{\xi}_{i}, t\right) \boldsymbol{I} \delta\left(\boldsymbol{\xi}-\boldsymbol{\xi}_{i}\right), \boldsymbol{H}_{b}(\boldsymbol{\xi}, t) \boldsymbol{\chi}(\boldsymbol{\xi}, t)=\sum_{i=1}^{M_{d}} \boldsymbol{H}_{d}\left(\boldsymbol{\xi}_{i}, t\right) \boldsymbol{\chi}\left(\boldsymbol{\xi}_{i}, t\right) \boldsymbol{I} \delta\left(\boldsymbol{\xi}-\boldsymbol{\xi}_{i}\right)
\end{aligned}
$$

the cost functional $L$ defined in (11) is changed to

$$
\begin{aligned}
L= & \frac{1}{2} \int_{D^{2}}\left\langle\left(\frac{\partial \boldsymbol{\chi}(\boldsymbol{x}, t)}{\partial t}-\boldsymbol{A}_{\boldsymbol{x}} \boldsymbol{\chi}(\boldsymbol{x}, t)\right), \boldsymbol{R}_{d}^{+}(\boldsymbol{x}, \boldsymbol{y}, t)\left(\frac{\partial \boldsymbol{\chi}(\boldsymbol{y}, t)}{\partial t}-\boldsymbol{A}_{\boldsymbol{y}} \boldsymbol{\chi}(\boldsymbol{y}, t)\right)\right\rangle d \boldsymbol{x} d \boldsymbol{y} \\
& +\frac{1}{2} \int_{S^{2}}\left\langle\boldsymbol{\beta}_{\boldsymbol{\xi}} \boldsymbol{\chi}(\boldsymbol{\xi}, t), \boldsymbol{R}_{b}^{+}(\boldsymbol{\xi}, \boldsymbol{\alpha}, t) \boldsymbol{\beta}_{\boldsymbol{\alpha}} \boldsymbol{\chi}(\boldsymbol{\alpha}, t)\right\rangle d S_{\boldsymbol{\xi}} d S_{\boldsymbol{\alpha}} \\
& +\frac{1}{2} \int_{D^{2}}\left\langle\sum_{i, j=1}^{M_{d}}\left[\boldsymbol{z}_{d}\left(\boldsymbol{x}_{i}, t\right)-\boldsymbol{H}_{d}\left(\boldsymbol{x}_{i}, t\right) \boldsymbol{\chi}\left(\boldsymbol{x}_{i}, t\right)\right], \boldsymbol{Q}_{d}^{+}\left(\boldsymbol{x}_{i}, \boldsymbol{y}_{j}, t\right)\right. \\
& \left.\times\left[\boldsymbol{z}_{d}\left(\boldsymbol{y}_{j}, t\right)-\boldsymbol{H}_{d}\left(\boldsymbol{y}_{j}, t\right) \boldsymbol{\chi}\left(\boldsymbol{y}_{j}, t\right)\right]\right\rangle \boldsymbol{I} \delta\left(\boldsymbol{x}-\boldsymbol{x}_{i}\right) \boldsymbol{I} \delta\left(\boldsymbol{y}-\boldsymbol{y}_{j}\right) d \boldsymbol{x} d \boldsymbol{y} \\
& +\frac{1}{2} \int_{S^{2}}\left\langle\sum_{i, j=1}^{M_{b}}\left[\boldsymbol{z}_{b}\left(\boldsymbol{\xi}_{i}, t\right)-\boldsymbol{H}_{b}\left(\boldsymbol{\xi}_{i}, t\right) \boldsymbol{\chi}\left(\boldsymbol{\xi}_{i}, t\right)\right], \boldsymbol{Q}_{b}^{+}\left(\boldsymbol{\xi}_{i}, \boldsymbol{\alpha}_{j}, t\right)\right. \\
& \left.\times\left[\boldsymbol{z}_{b}\left(\boldsymbol{\alpha}_{j}, t\right)-\boldsymbol{H}_{b}\left(\boldsymbol{\alpha}_{j}, t\right) \boldsymbol{\chi}\left(\boldsymbol{\alpha}_{j}, t\right)\right]\right\rangle \boldsymbol{I} \delta\left(\boldsymbol{\xi}-\boldsymbol{\xi}_{i}\right) \boldsymbol{I} \delta\left(\boldsymbol{\alpha}-\boldsymbol{\alpha}_{j}\right) d S_{\boldsymbol{\xi}} d S_{\boldsymbol{\alpha}},
\end{aligned}
$$


Following the same procedure as in the previous subsection with the cost functional $L$ given in (58) yields the following observer

$$
\begin{aligned}
& \frac{\partial \hat{\boldsymbol{\chi}}(\boldsymbol{x}, t)}{\partial t}=\boldsymbol{A}_{\boldsymbol{x}} \hat{\boldsymbol{\chi}}(\boldsymbol{x}, t) \\
& \quad+\sum_{i, j=1}^{M_{d}} \boldsymbol{P}\left(\boldsymbol{x}, \boldsymbol{y}_{i}, t\right) \boldsymbol{H}_{d}^{T}\left(\boldsymbol{y}_{i}, t\right) \boldsymbol{Q}_{d}^{+}\left(\boldsymbol{y}_{i}, \boldsymbol{y}_{j}, t\right)\left[\boldsymbol{z}_{d}\left(\boldsymbol{y}_{j}, t\right)-\boldsymbol{H}_{d}\left(\boldsymbol{y}_{j}, t\right) \hat{\boldsymbol{\chi}}\left(\boldsymbol{y}_{j}, t\right)\right] \\
& \quad+\sum_{i, j=1}^{M_{b}} \boldsymbol{P}\left(\boldsymbol{x}, \boldsymbol{\xi}_{i}, t\right) \boldsymbol{H}_{b}^{T}\left(\boldsymbol{\xi}_{i}, t\right) \boldsymbol{Q}_{b}^{+}\left(\boldsymbol{\xi}_{i}, \boldsymbol{\alpha}_{j}, t\right)\left[\boldsymbol{z}_{b}\left(\boldsymbol{\alpha}_{j}, t\right)-\boldsymbol{H}_{b}\left(\boldsymbol{\alpha}_{j}, t\right) \hat{\chi}\left(\boldsymbol{\alpha}_{j}, t\right)\right], \\
& \hat{\boldsymbol{\chi}}\left(\boldsymbol{x}, t_{0}\right)=\chi_{0}(\boldsymbol{x}), \boldsymbol{\beta}_{\boldsymbol{\xi}} \hat{\boldsymbol{\chi}}(\boldsymbol{\xi}, t)=0
\end{aligned}
$$

where $\boldsymbol{P}(\boldsymbol{x}, \boldsymbol{y}, t)$ is generated by

$$
\begin{aligned}
\frac{\partial \boldsymbol{P}(\boldsymbol{x}, \boldsymbol{y}, t)}{\partial t} & =\overline{\boldsymbol{A}}_{\boldsymbol{x}} \boldsymbol{P}(\boldsymbol{x}, \boldsymbol{y}, t)-\sum_{i, j=1}^{M_{d}} \boldsymbol{P}\left(\boldsymbol{x}, \boldsymbol{y}_{i}, t\right) \overline{\boldsymbol{Q}}_{d}\left(\boldsymbol{y}_{i}, \boldsymbol{y}_{j}, t\right) \boldsymbol{P}\left(\boldsymbol{y}_{j}, \boldsymbol{y}, t\right) \\
& +\boldsymbol{P}(\boldsymbol{x}, \boldsymbol{y}, t) \overline{\boldsymbol{A}}_{\boldsymbol{y}}^{T}-\sum_{i, j=1}^{M_{b}} \boldsymbol{P}\left(\boldsymbol{x}, \boldsymbol{\xi}_{i}, t\right) \overline{\boldsymbol{Q}}_{b}\left(\boldsymbol{\xi}_{i}, \boldsymbol{\alpha}_{j}, t\right) \boldsymbol{P}\left(\boldsymbol{\alpha}_{j}, \boldsymbol{y}, t\right), \\
\boldsymbol{P}\left(\boldsymbol{x}, \boldsymbol{y}, t_{0}\right) & =\boldsymbol{P}_{0}(\boldsymbol{x}, \boldsymbol{y}), \overline{\boldsymbol{\beta}}_{\boldsymbol{\xi}} \boldsymbol{P}(\boldsymbol{\xi}, \boldsymbol{y}, t)=0,
\end{aligned}
$$

which has a very similar structure with the observer (49) and (50) but the distributed measurements are replaced by the spatial discrete ones.

The observer error dynamics are obtained by differentiating both sides of the first equation of (51) with respect to $t$ along the solutions of the observer system (59), the original system (1), and the measurement system (56) as follows

$$
\begin{aligned}
& \frac{\partial \boldsymbol{\chi}_{e}(\boldsymbol{x}, t)}{\partial t}=\boldsymbol{A}_{\boldsymbol{x}} \boldsymbol{\chi}_{e}(\boldsymbol{x}, t)+\boldsymbol{w}_{d}(\boldsymbol{x}, t) \\
& \quad-\sum_{i, j=1}^{M_{d}} \boldsymbol{P}\left(\boldsymbol{x}, \boldsymbol{y}_{i}, t\right)\left(\overline{\boldsymbol{Q}}_{d}\left(\boldsymbol{y}_{i}, \boldsymbol{y}_{j}, t\right) \boldsymbol{\chi}_{e}\left(\boldsymbol{y}_{j}, t\right)+\boldsymbol{H}_{d}^{T}\left(\boldsymbol{y}_{i}, t\right) \boldsymbol{Q}_{d}^{+}\left(\boldsymbol{y}_{i}, \boldsymbol{y}_{j}, t\right) \boldsymbol{\varepsilon}_{d}\left(\boldsymbol{y}_{j}, t\right)\right) \\
& \quad-\sum_{i, j=1}^{M_{b}} \boldsymbol{P}\left(\boldsymbol{x}, \boldsymbol{\xi}_{i}, t\right)\left(\overline{\boldsymbol{Q}}_{b}\left(\boldsymbol{\xi}_{i}, \boldsymbol{\alpha}_{j}, t\right) \boldsymbol{\chi}_{e}\left(\boldsymbol{\alpha}_{j}, t\right)+\boldsymbol{H}_{b}^{T}\left(\boldsymbol{\xi}_{i}, t\right) \boldsymbol{Q}_{b}^{+}\left(\boldsymbol{\xi}_{i}, \boldsymbol{\alpha}_{j}, t\right) \boldsymbol{\varepsilon}_{b}\left(\boldsymbol{\alpha}_{j}, t\right)\right), \\
& \boldsymbol{\chi}_{e}\left(\boldsymbol{x}, t_{0}\right)=\boldsymbol{w}_{0}(\boldsymbol{x}), \boldsymbol{\beta}_{\boldsymbol{\xi}} \boldsymbol{\chi}_{e}(\boldsymbol{\xi}, t)=\boldsymbol{w}_{b}(\boldsymbol{\xi}, t) .
\end{aligned}
$$

\subsubsection{Lyapunov stability analysis}

To analyze stability of the observer error system (61) at the origin, we still consider the Lyapunov functional candidate (53). Differentiating both sides of (53) with 
respect time $t$ along the solutions of (61) yields

$$
\begin{aligned}
& \frac{d V(t)}{d t}=-\sum_{i, j=1}^{M_{d}} \boldsymbol{\chi}_{e}\left(\boldsymbol{x}_{i}, t\right), \overline{\boldsymbol{Q}}_{d}\left(\boldsymbol{x}_{i}, \boldsymbol{y}_{j}, t\right) \boldsymbol{\chi}_{e}\left(\boldsymbol{y}_{j}, t\right)-\sum_{i, j=1}^{M_{b}} \boldsymbol{\chi}_{e}\left(\boldsymbol{\xi}_{i}, t\right), \overline{\boldsymbol{Q}}_{b}\left(\boldsymbol{\xi}_{i}, \boldsymbol{\alpha}_{j}, t\right) \boldsymbol{\chi}_{e}\left(\boldsymbol{y}_{j}, t\right) \\
& -\int_{D^{2}}\left\langle\boldsymbol{\chi}_{e}(\boldsymbol{x}, t), \overline{\boldsymbol{R}}_{d}(\boldsymbol{x}, \boldsymbol{y}, t) \boldsymbol{\chi}_{e}(\boldsymbol{y}, t)\right\rangle d \boldsymbol{x} d \boldsymbol{y}+2 \int_{D^{2}}\left\langle\boldsymbol{w}_{d}(\boldsymbol{x}, t), \boldsymbol{P}^{+}(\boldsymbol{x}, \boldsymbol{y}, t) \boldsymbol{\chi}_{e}(\boldsymbol{y}, t)\right\rangle d \boldsymbol{x} d \boldsymbol{y} \\
& -2 \sum_{i, j=1}^{M_{d}}\left\langle\boldsymbol{\varepsilon}_{d}\left(\boldsymbol{x}_{i}, t\right), \boldsymbol{Q}_{d}^{+}\left(\boldsymbol{x}_{i}, \boldsymbol{y}_{j}, t\right) \boldsymbol{H}_{d}\left(\boldsymbol{y}_{j}, t\right) \boldsymbol{\chi}_{e}\left(\boldsymbol{y}_{j}, t\right)\right\rangle \\
& -2 \int_{S^{2}}\left\langle\varepsilon_{b}(\boldsymbol{\xi}, t), \boldsymbol{Q}_{b}^{+}(\boldsymbol{\xi}, \boldsymbol{\alpha}, t) \boldsymbol{H}_{b}(\boldsymbol{\alpha}, t) \boldsymbol{\chi}_{e}(\boldsymbol{\alpha}, t)\right\rangle d S_{\boldsymbol{\alpha}} d S_{\boldsymbol{\xi}} .
\end{aligned}
$$

Convergence of $\boldsymbol{\chi}_{e}(\boldsymbol{x}, t)$ and $\boldsymbol{\chi}_{e}(\boldsymbol{\xi}, t)$ to a ball centered at the origin can be analyzed using the same arguments as in Subsection 4.2.2 with a note that one should apply the formula (7) to the last integral term in the right hand side of (62).

\section{Conclusions}

The calculus of variation approach was used to propose a constructive method to design inverse optimal filters for a class of linear distributed parameter systems. The systems can contain disturbances distributed over the interior and the boundary. Moreover, both spatial continuous and discrete measurements corrupted by disturbances were addressed. The most shining point of the paper is the introduction of inverse optimality concept that relaxes difficulties in solving the Riccati nonlinear PDEs. Novel fundamental results, e.g., the Green matrix formula, the derivation of unique and bounded solution of a linear PDE, and the analytical solution of a Bernoulli PDE, on linear and nonlinear PDEs developed in this paper can be further used for solving other filter design problems for distributed parameter systems and further improve performance of controlling practical distributed parameter systems as as marine riser systems in [4], [5], [16].

\section{A Proof of Lemma 3.1}

From definition of $\boldsymbol{A}_{\boldsymbol{x}}$ in (3) and $\boldsymbol{A}_{\boldsymbol{x}}^{*}$ in (13), we have

$$
\begin{aligned}
& \left\langle\boldsymbol{\lambda}, \boldsymbol{A}_{\boldsymbol{x}} \boldsymbol{\gamma}\right\rangle-\left\langle\boldsymbol{\gamma}, \boldsymbol{A}_{\boldsymbol{x}}^{*} \boldsymbol{\lambda}\right\rangle=\sum_{i, j=1}^{n}\left[\left\langle\boldsymbol{\lambda}, \boldsymbol{A}_{i j} \frac{\partial^{2} \boldsymbol{\gamma}}{\partial x_{i} \partial x_{j}}\right\rangle-\left\langle\frac{\partial^{2}\left(\boldsymbol{A}_{i j} \boldsymbol{\lambda}\right)}{\partial x_{i} \partial x_{j}}, \boldsymbol{\gamma}\right\rangle\right] \\
& +\sum_{i}^{n}\left[\left\langle\boldsymbol{\lambda}, \boldsymbol{B}_{i} \frac{\partial \boldsymbol{\gamma}}{\partial x_{i}}\right\rangle+\left\langle\frac{\partial\left(\boldsymbol{B}_{i} \boldsymbol{\lambda}\right)}{\partial x_{i}}, \boldsymbol{\gamma}\right\rangle\right] \\
& =\sum_{i=1}^{n} \frac{\partial}{\partial x_{i}}\left[\sum_{j=1}^{n}\left(\left\langle\frac{\partial \boldsymbol{\gamma}}{\partial x_{j}}, \boldsymbol{A}_{i j} \boldsymbol{\lambda}\right\rangle-\left\langle\boldsymbol{\gamma}, \frac{\partial\left(\boldsymbol{A}_{i j} \boldsymbol{\lambda}\right)}{\partial x_{j}}\right\rangle\right)+\left\langle\boldsymbol{\gamma}, \boldsymbol{B}_{i} \boldsymbol{\lambda}\right\rangle\right]:=\sum_{i=1}^{n} \frac{\partial \boldsymbol{H}_{i}}{\partial x_{i}}
\end{aligned}
$$


where

$$
\boldsymbol{H}_{i}=\sum_{j=1}^{n}\left(\left\langle\boldsymbol{A}_{i j} \frac{\partial \boldsymbol{\gamma}}{\partial x_{j}}, \boldsymbol{\lambda}\right\rangle-\left\langle\boldsymbol{\gamma}, \boldsymbol{A}_{i j} \frac{\partial \boldsymbol{\lambda}}{\partial x_{j}}\right\rangle-\left\langle\boldsymbol{\gamma}, \frac{\partial \boldsymbol{A}_{i j}}{\partial x_{j}} \boldsymbol{\lambda}\right\rangle\right)+\left\langle\boldsymbol{\gamma}, \boldsymbol{B}_{i} \boldsymbol{\lambda}\right\rangle .
$$

On the other hand, from the Gauss theorem [13] we have

$$
\int_{D} \sum_{i=1}^{n} \frac{\partial \boldsymbol{H}_{i}}{\partial x_{i}} d \boldsymbol{x}=\int_{S} \sum_{i=1}^{n} \boldsymbol{H}_{i}(\boldsymbol{\xi}, t) \cos \left(\boldsymbol{n}_{\boldsymbol{\xi}}, x_{i}\right) d S_{\boldsymbol{\xi}}
$$

Integrating both sides of (63) over $D$ and using (65) give

$$
\int_{D}\left\langle\boldsymbol{\lambda}, \boldsymbol{A}_{\boldsymbol{x}} \boldsymbol{\gamma}\right\rangle-\left\langle\boldsymbol{\gamma}, \boldsymbol{A}_{\boldsymbol{x}}^{*} \boldsymbol{\lambda}\right\rangle d \boldsymbol{x}=\int \sum_{D_{i=1}}^{n} \frac{\partial \boldsymbol{H}_{i}}{\partial x_{i}} d \boldsymbol{x}=\int \sum_{S_{i=1}^{n}}^{n} \boldsymbol{H}_{i}(\boldsymbol{\xi}, t) \cos \left(\boldsymbol{n}_{\boldsymbol{\xi}}, x_{i}\right) d S_{\boldsymbol{\xi}}
$$

Substituting the expression of $\boldsymbol{H}_{i}(\boldsymbol{x}, t)$ defined in (64) with $\boldsymbol{x}$ replaced by $\boldsymbol{\xi}$ into (66) results in

$$
\begin{array}{r}
\int_{D}\left\langle\boldsymbol{\lambda}, \boldsymbol{A}_{\boldsymbol{x}} \boldsymbol{\gamma}\right\rangle-\left\langle\boldsymbol{\gamma}, \boldsymbol{A}_{\boldsymbol{x}}^{*} \boldsymbol{\lambda}\right\rangle d \boldsymbol{x}=\int_{S} \sum_{i=1}^{n}\left[\sum _ { j = 1 } ^ { n } \left(\left\langle\boldsymbol{A}_{i j} \frac{\partial \boldsymbol{\gamma}}{\partial x_{j}}, \boldsymbol{\lambda}\right\rangle-\right.\right. \\
\left.\left.\left\langle\boldsymbol{\gamma}, \boldsymbol{A}_{i j} \frac{\partial \boldsymbol{\lambda}}{\partial x_{j}}\right\rangle-\left\langle\boldsymbol{\gamma}, \frac{\partial \boldsymbol{A}_{i j}}{\partial x_{j}} \boldsymbol{\lambda}\right\rangle\right)+\left\langle\boldsymbol{\gamma}, \boldsymbol{B}_{i} \boldsymbol{\lambda}\right\rangle\right]\left.\right|_{\boldsymbol{x}=\boldsymbol{\xi}} \cos \left(\boldsymbol{n}_{\boldsymbol{\xi}}, x_{i}\right) d S_{\boldsymbol{\xi}}
\end{array}
$$

Expanding the right hand side of (67) gives

$$
\begin{aligned}
\int_{D}\left\langle\boldsymbol{\lambda}, \boldsymbol{A}_{\boldsymbol{x}} \boldsymbol{\gamma}\right\rangle-\left\langle\boldsymbol{\gamma}, \boldsymbol{A}_{\boldsymbol{x}}^{*} \boldsymbol{\lambda}\right\rangle d \boldsymbol{x}= \\
\int_{S} \sum_{j=1}^{n}\left[\sum_{i=1}^{n}\left\langle\boldsymbol{A}_{i j}(\boldsymbol{\xi}, t) \frac{\partial \boldsymbol{\gamma}(\boldsymbol{\xi}, t)}{\partial x_{j}} \cos \left(\boldsymbol{n}_{\boldsymbol{\xi}}, x_{i}\right), \boldsymbol{\lambda}(\boldsymbol{\xi}, t)\right\rangle\right] d S_{\boldsymbol{\xi}}- \\
\int_{S} \sum_{j=1}^{n}\left[\sum_{i=1}^{n}\left\langle\gamma(\boldsymbol{\xi}, t), \boldsymbol{A}_{i j}(\boldsymbol{\xi}, t) \frac{\partial \boldsymbol{\lambda}(\boldsymbol{\xi}, t)}{\partial x_{j}} \cos \left(\boldsymbol{n}_{\boldsymbol{\xi}}, x_{i}\right)\right\rangle\right] d S_{\boldsymbol{\xi}}+ \\
\int_{S} \sum_{i=1}^{n}\left\langle\boldsymbol{\gamma}(\boldsymbol{\xi}, t),\left(\boldsymbol{B}_{i}(\boldsymbol{\xi}, t)-\sum_{j=1}^{n} \frac{\partial \boldsymbol{A}_{i j}(\boldsymbol{\xi}, t)}{\partial x_{j}}\right) \cos \left(\boldsymbol{n}_{\boldsymbol{\xi}}, x_{i}\right) \boldsymbol{\lambda}(\boldsymbol{\xi}, t)\right\rangle d S_{\boldsymbol{\xi}}
\end{aligned}
$$

Substituting the definition of $\boldsymbol{A}_{j}(\boldsymbol{\xi}, t)$ and $\boldsymbol{K}(\boldsymbol{\xi}, t)$ defined in (4) and (14), respectively, into (68)

$$
\begin{aligned}
& \int_{D}\left\langle\boldsymbol{\lambda}, \boldsymbol{A}_{\boldsymbol{x}} \boldsymbol{\gamma}\right\rangle-\left\langle\boldsymbol{\gamma}, \boldsymbol{A}_{\boldsymbol{x}}^{*} \boldsymbol{\lambda}\right\rangle d \boldsymbol{x}=\int_{S} \sum_{j=1}^{n}\left\langle\boldsymbol{A}_{j}(\boldsymbol{\xi}, t) \frac{\partial \boldsymbol{\gamma}(\boldsymbol{\xi}, t)}{\partial x_{j}}, \boldsymbol{\lambda}(\boldsymbol{\xi}, t)\right\rangle d S_{\boldsymbol{\xi}} \\
& -\int_{S} \sum_{j=1}^{n}\left\langle\boldsymbol{\gamma}(\boldsymbol{\xi}, t), \boldsymbol{A}_{j}(\boldsymbol{\xi}, t) \frac{\partial \boldsymbol{\lambda}(\boldsymbol{\xi}, t)}{\partial x_{j}}\right\rangle d S_{\boldsymbol{\xi}}+\int_{S} \sum_{i=1}^{n}\langle\boldsymbol{\gamma}(\boldsymbol{\xi}, t), \boldsymbol{K}(\boldsymbol{\xi}, t) \boldsymbol{\lambda}(\boldsymbol{\xi}, t)\rangle d S_{\boldsymbol{\xi}}
\end{aligned}
$$

Adding and subtracting $\int_{S}\langle\boldsymbol{\gamma}(\boldsymbol{\xi}, t), \boldsymbol{F}(\boldsymbol{\xi}, t) \boldsymbol{\lambda}(\boldsymbol{\xi}, t)\rangle d S_{\boldsymbol{\xi}}$ to the right hand side of (69) yields (12) by interchanging the role of $\boldsymbol{\gamma}(\boldsymbol{\xi}, t)$ and $\boldsymbol{\lambda}(\boldsymbol{\xi}, t)$ in $\boldsymbol{\beta}_{\boldsymbol{\xi}}(\boldsymbol{\xi}, t) \boldsymbol{\gamma}(\boldsymbol{\xi}, t)$ and $\boldsymbol{\beta}_{\boldsymbol{\xi}}^{*}(\boldsymbol{\xi}, t) \boldsymbol{\gamma}(\boldsymbol{\xi}, t)$, which are given in (3) and (13), respectively. 


\section{B Proof of Lemma 3.2}

Differentiating both sides of (15) with respect to $t$ gives

$$
\int_{D} \frac{\partial \boldsymbol{A}\left(\boldsymbol{x}^{\prime}, \boldsymbol{y}^{\prime}, t\right)}{\partial t} \boldsymbol{A}^{+}\left(\boldsymbol{y}^{\prime}, \boldsymbol{y}, t\right) d \boldsymbol{y}^{\prime}+\int_{D} \boldsymbol{A}\left(\boldsymbol{x}^{\prime}, \boldsymbol{y}^{\prime}, t\right) \frac{\partial \boldsymbol{A}^{+}\left(\boldsymbol{y}^{\prime}, \boldsymbol{y}, t\right)}{\partial t} d \boldsymbol{y}^{\prime}=0 .
$$

Multiplying both sides of (70) with $\boldsymbol{A}^{+}\left(\boldsymbol{x}, \boldsymbol{x}^{\prime}, t\right)$ then integrating over $D$ yield

$$
\begin{aligned}
\int_{D^{2}} \boldsymbol{A}^{+}\left(\boldsymbol{x}, \boldsymbol{x}^{\prime}, t\right) \frac{\partial \boldsymbol{A}\left(\boldsymbol{x}^{\prime}, \boldsymbol{y}^{\prime}, t\right)}{\partial t} \boldsymbol{A}^{+}\left(\boldsymbol{y}^{\prime}, \boldsymbol{y}, t\right) d \boldsymbol{y}^{\prime} d \boldsymbol{x}^{\prime} \\
+\int_{D^{2}} \boldsymbol{A}^{+}\left(\boldsymbol{x}, \boldsymbol{x}^{\prime}, t\right) \boldsymbol{A}\left(\boldsymbol{x}^{\prime}, \boldsymbol{y}^{\prime}, t\right) \frac{\partial \boldsymbol{A}^{+}\left(\boldsymbol{y}^{\prime}, \boldsymbol{y}, t\right)}{\partial t} d \boldsymbol{y}^{\prime} d \boldsymbol{x}^{\prime}=0 .
\end{aligned}
$$

Substituting $\int_{D} \boldsymbol{A}^{+}\left(\boldsymbol{x}, \boldsymbol{x}^{\prime}, t\right) \boldsymbol{A}\left(\boldsymbol{x}^{\prime}, \boldsymbol{y}^{\prime}, t\right) \boldsymbol{x}^{\prime}=\boldsymbol{I} \delta\left(\boldsymbol{x}-\boldsymbol{y}^{\prime}\right)$ into (71) results in

$$
\int_{D^{2}} \boldsymbol{A}^{+}\left(\boldsymbol{x}, \boldsymbol{x}^{\prime}, t\right) \frac{\partial \boldsymbol{A}\left(\boldsymbol{x}^{\prime}, \boldsymbol{y}^{\prime}, t\right)}{\partial t} \boldsymbol{A}^{+}\left(\boldsymbol{y}^{\prime}, \boldsymbol{y}, t\right) d \boldsymbol{y}^{\prime} d \boldsymbol{x}^{\prime}+\int_{D} \boldsymbol{I} \delta\left(\boldsymbol{x}-\boldsymbol{y}^{\prime}\right) \frac{\partial \boldsymbol{A}^{+}\left(\boldsymbol{y}^{\prime}, \boldsymbol{y}, t\right)}{\partial t} d \boldsymbol{y}^{\prime}=0
$$

which is (16) since $\int_{D} \boldsymbol{I} \delta\left(\boldsymbol{x}-\boldsymbol{y}^{\prime}\right) \frac{\partial \boldsymbol{A}^{+}\left(\boldsymbol{y}^{\prime}, \boldsymbol{y}, t\right)}{\partial t} d \boldsymbol{y}^{\prime}=\frac{\partial \boldsymbol{A}^{+}(\boldsymbol{x}, \boldsymbol{y}, t)}{\partial t}$.

\section{Proof of Lemma 3.3}

\section{C.1 Verification of the solution $\boldsymbol{N}(\boldsymbol{x}, \boldsymbol{y}, t)$}

\section{C.1.1 Verification of the initial condition and boundary condition} From (18), we have

$$
\begin{aligned}
\boldsymbol{N}\left(\boldsymbol{x}, \boldsymbol{y}, t_{0}\right) & =\int_{D^{2}} \boldsymbol{G}\left(\boldsymbol{x}, t_{0} ; \boldsymbol{x}^{\prime}, t_{0}\right) \boldsymbol{N}_{0}\left(\boldsymbol{x}^{\prime}, \boldsymbol{y}^{\prime}\right) \boldsymbol{G}^{T}\left(\boldsymbol{y}, t_{0} ; \boldsymbol{y}^{\prime}, t_{0}\right) d \boldsymbol{x}^{\prime} d \boldsymbol{y}^{\prime} \\
& =\int_{D^{2}} \boldsymbol{I} \delta\left(\boldsymbol{x}-\boldsymbol{x}^{\prime}\right) \boldsymbol{N}_{0}\left(\boldsymbol{x}^{\prime}, \boldsymbol{y}^{\prime}\right) \boldsymbol{I} \delta\left(\boldsymbol{y}-\boldsymbol{y}^{\prime}\right) d \boldsymbol{x}^{\prime} d \boldsymbol{y}^{\prime}=\boldsymbol{N}_{0}(\boldsymbol{x}, \boldsymbol{y}),
\end{aligned}
$$

where we have used the second equation of (19). Thus the initial condition is verified. Also, from (18), we have

$$
\begin{aligned}
& \overline{\boldsymbol{\beta}}_{\boldsymbol{\xi}} \boldsymbol{N}(\boldsymbol{\xi}, \boldsymbol{y}, t)=\int_{D^{2}} \overline{\boldsymbol{\beta}}_{\boldsymbol{\xi}} \boldsymbol{G}\left(\boldsymbol{\xi}, t ; \boldsymbol{x}^{\prime}, t_{0}\right) \boldsymbol{N}_{0}\left(\boldsymbol{x}^{\prime}, \boldsymbol{y}^{\prime}\right) \boldsymbol{G}^{T}\left(\boldsymbol{y}, t ; \boldsymbol{y}^{\prime}, t_{0}\right) d \boldsymbol{x}^{\prime} d \boldsymbol{y}^{\prime}+ \\
& \int_{t_{0}}^{t} \int_{D^{2}} \overline{\boldsymbol{\beta}}_{\boldsymbol{\xi}} \boldsymbol{G}\left(\boldsymbol{\xi}, t-\tau+t_{0} ; \boldsymbol{x}^{\prime}, t_{0}\right) \boldsymbol{R}\left(\boldsymbol{x}^{\prime}, \boldsymbol{y}^{\prime}, \tau\right) \boldsymbol{G}^{T}\left(\boldsymbol{y}, t-\tau+t_{0} ; \boldsymbol{y}^{\prime}, t_{0}\right) d \boldsymbol{x}^{\prime} d \boldsymbol{y}^{\prime} d \tau=0
\end{aligned}
$$

where we have used the third equation of (19). Thus the boundary condition is verified. 
C.1.2 Verification of $\boldsymbol{N}(\boldsymbol{x}, \boldsymbol{y}, t)$ satisfied the first equation of (17)

Differentiating both sides of (18) with respect to $t$ gives

$$
\begin{aligned}
& \frac{\partial \boldsymbol{N}(\boldsymbol{x}, \boldsymbol{y}, t)}{\partial t}=-\int_{D^{2}} \overline{\boldsymbol{A}}_{\boldsymbol{x}} \boldsymbol{G}\left(\boldsymbol{x}, t ; \boldsymbol{x}^{\prime}, t_{0}\right) \boldsymbol{N}_{0}\left(\boldsymbol{x}^{\prime}, \boldsymbol{y}^{\prime}\right) \boldsymbol{G}^{T}\left(\boldsymbol{y}, t ; \boldsymbol{y}^{\prime}, t_{0}\right) d \boldsymbol{x}^{\prime} d \boldsymbol{y}^{\prime} \\
& -\int_{t_{0}}^{t} \int_{D^{2}} \overline{\boldsymbol{A}}_{\boldsymbol{x}} \boldsymbol{G}\left(\boldsymbol{x}, t-\tau+t_{0} ; \boldsymbol{x}^{\prime}, t_{0}\right) \boldsymbol{R}\left(\boldsymbol{x}^{\prime}, \boldsymbol{y}^{\prime}, \tau\right) \boldsymbol{G}^{T}\left(\boldsymbol{y}, t-\tau+t_{0} ; \boldsymbol{y}^{\prime}, t_{0}\right) d \boldsymbol{x}^{\prime} d \boldsymbol{y}^{\prime} d \tau \\
& -\int_{D^{2}} \boldsymbol{G}\left(\boldsymbol{x}, t ; \boldsymbol{x}^{\prime}, t_{0}\right) \boldsymbol{N}_{0}\left(\boldsymbol{x}^{\prime}, \boldsymbol{y}^{\prime}\right) \boldsymbol{G}^{T}\left(\boldsymbol{y}, t ; \boldsymbol{y}^{\prime}, t_{0}\right) \overline{\boldsymbol{A}}_{\boldsymbol{y}}^{T} d \boldsymbol{x}^{\prime} d \boldsymbol{y}^{\prime} \\
& -\int_{t_{0}}^{t} \int_{D^{2}} \boldsymbol{G}\left(\boldsymbol{x}, t-\tau+t_{0} ; \boldsymbol{x}^{\prime}, t_{0}\right) \boldsymbol{R}\left(\boldsymbol{x}^{\prime}, \boldsymbol{y}^{\prime}, \tau\right) \boldsymbol{G}^{T}\left(\boldsymbol{y}, t-\tau+t_{0} ; \boldsymbol{y}^{\prime}, t_{0}\right) \overline{\boldsymbol{A}}_{\boldsymbol{y}}^{T} d \boldsymbol{x}^{\prime} d \boldsymbol{y}^{\prime} d \tau \\
& +\int_{D^{2}} \boldsymbol{I} \delta\left(\boldsymbol{x}-\boldsymbol{x}^{\prime}\right) \boldsymbol{R}\left(\boldsymbol{x}^{\prime}, \boldsymbol{y}^{\prime}, t\right) \boldsymbol{I} \delta(\boldsymbol{y}-\boldsymbol{y}) d \boldsymbol{x}^{\prime} d \boldsymbol{y}^{\prime} \\
= & -\overline{\boldsymbol{A}}_{\boldsymbol{x}} \boldsymbol{N}(\boldsymbol{x}, \boldsymbol{y}, t)-\boldsymbol{N}(\boldsymbol{x}, \boldsymbol{y}, t) \overline{\boldsymbol{A}}_{\boldsymbol{y}}^{T}+\boldsymbol{R}(\boldsymbol{x}, \boldsymbol{y}, t),
\end{aligned}
$$

where we have used the first two equations of (19). Thus the solution $\boldsymbol{N}(\boldsymbol{x}, \boldsymbol{y}, t)$ satisfies the first equation of (17).

\section{C.2 Proof of uniqueness of $\boldsymbol{N}(\boldsymbol{x}, \boldsymbol{y}, t)$}

Assume that there are two solutions $\boldsymbol{N}_{1}(\boldsymbol{x}, \boldsymbol{y}, t)$ and $\boldsymbol{N}_{2}(\boldsymbol{x}, \boldsymbol{y}, t)$ satisfy (17). Let $\boldsymbol{N}_{e}(\boldsymbol{x}, \boldsymbol{y}, t)=\boldsymbol{N}_{1}(\boldsymbol{x}, \boldsymbol{y}, t)-\boldsymbol{N}_{2}(\boldsymbol{x}, \boldsymbol{y}, t)$. Then, substituting $\boldsymbol{N}_{1}(\boldsymbol{x}, \boldsymbol{y}, t)$ and $\boldsymbol{N}_{2}(\boldsymbol{x}, \boldsymbol{y}, t)$ then subtracting from each other yield

$$
\begin{aligned}
& \frac{\partial \boldsymbol{N}_{e}(\boldsymbol{x}, \boldsymbol{y}, t)}{\partial t}=-\overline{\boldsymbol{A}}_{\boldsymbol{x}} \boldsymbol{N}_{e}(\boldsymbol{x}, \boldsymbol{y}, t)-\boldsymbol{N}_{e}(\boldsymbol{x}, \boldsymbol{y}, t) \overline{\boldsymbol{A}}_{\boldsymbol{y}}^{T}, \\
& \boldsymbol{N}_{e}\left(\boldsymbol{x}, \boldsymbol{y}, t_{0}\right)=0, \\
& \overline{\boldsymbol{\beta}}_{\boldsymbol{\xi}} \boldsymbol{N}_{e}(\boldsymbol{\xi}, \boldsymbol{y}, t)=0, \quad \boldsymbol{\xi} \in S, \boldsymbol{y} \in D .
\end{aligned}
$$

Applying (74) to (76) yields $\boldsymbol{N}_{e}(\boldsymbol{x}, \boldsymbol{y}, t)=0$. Hence $\boldsymbol{N}_{1}(\boldsymbol{x}, \boldsymbol{y}, t)=\boldsymbol{N}_{2}(\boldsymbol{x}, \boldsymbol{y}, t)$, i.e., the solution $\boldsymbol{N}(\boldsymbol{x}, \boldsymbol{y}, t)$ defined in (18) is the unique solution of (17).

\section{Proof of Lemma 3.4}

To prove Lemma 3.4, we first verify that $\boldsymbol{P}(\boldsymbol{x}, \boldsymbol{y}, t)$ given in (21) satisfies the Riccati nonlinear partial differential equation (20). Then, we prove that the solution $\boldsymbol{P}(\boldsymbol{x}, \boldsymbol{y}, t)$ given in $(21)$ is unique and bounded.

\section{D.1 Verification of the solution $\boldsymbol{P}(\boldsymbol{x}, \boldsymbol{y}, t)$}

We proceed by proving that $\boldsymbol{P}(\boldsymbol{x}, \boldsymbol{y}, t)$ satisfies the terminal condition, i.e., the second equation in (20), the boundary condition i.e., the third equation in (20), and the first equation (20). 
First, to prove that $\boldsymbol{P}(\boldsymbol{x}, \boldsymbol{y}, t)$ satisfies the terminal condition in (20), from (22) and (23), we have

$$
\boldsymbol{M}\left(\boldsymbol{x}, \boldsymbol{y}, t_{0}\right)=0, \boldsymbol{G}\left(\boldsymbol{x}, t_{0} ; \boldsymbol{x}^{\prime}, t_{0}\right)=\boldsymbol{I} \delta\left(\boldsymbol{x}-\boldsymbol{x}^{\prime}\right) .
$$

Substituting (77) into the third equation of (21) at $t=t_{0}$ yields

$$
\boldsymbol{P}\left(\boldsymbol{x}, \boldsymbol{y}, t_{0}\right)=\int_{D^{2}} \boldsymbol{I} \delta\left(\boldsymbol{x}-\boldsymbol{x}^{\prime}\right) \boldsymbol{P}_{0}\left(\boldsymbol{x}^{\prime}, \boldsymbol{y}^{\prime}\right) \boldsymbol{I} \delta\left(\boldsymbol{y}-\boldsymbol{y}^{\prime}\right) d \boldsymbol{x}^{\prime} d \boldsymbol{y}^{\prime}=\boldsymbol{P}_{0}(\boldsymbol{x}, \boldsymbol{y}) .
$$

Second, to prove that $\boldsymbol{P}(\boldsymbol{x}, \boldsymbol{y}, t)$ satisfies the boundary condition in (20), from the third equation of (21), we have

$\overline{\boldsymbol{\beta}}_{\boldsymbol{\xi}} \boldsymbol{P}(\boldsymbol{\xi}, \boldsymbol{y}, t)=\int_{D^{2}} \overline{\boldsymbol{\beta}}_{\boldsymbol{\xi}} \boldsymbol{G}\left(\boldsymbol{\xi}, t ; \boldsymbol{x}^{\prime}, t_{0}\right)\left[\boldsymbol{P}_{0}^{+}\left(\boldsymbol{x}^{\prime}, \boldsymbol{y}^{\prime}\right)+\boldsymbol{M}\left(\boldsymbol{x}^{\prime}, \boldsymbol{y}^{\prime}, t\right)\right]^{+} \boldsymbol{G}^{T}\left(\boldsymbol{y}, t ; \boldsymbol{y}^{\prime}, t_{0}\right) d \boldsymbol{x}^{\prime} d \boldsymbol{y}^{\prime}=0$

where we have used the third equation of (23).

Third, to prove that $\boldsymbol{P}(\boldsymbol{x}, \boldsymbol{y}, t)$ satisfies the first equation of (20), we differentiating both sides of the third equation of (21) with respect to $\bar{t}$ to obtain

$$
\begin{aligned}
& \frac{\partial \boldsymbol{P}(\boldsymbol{x}, \boldsymbol{y}, t)}{\partial t}=\int_{D^{2}} \frac{\partial \boldsymbol{G}\left(\boldsymbol{x}, t ; \boldsymbol{x}^{\prime}, t_{0}\right)}{\partial t}\left[\boldsymbol{P}_{0}^{+}\left(\boldsymbol{x}^{\prime}, \boldsymbol{y}^{\prime}\right)+\boldsymbol{M}\left(\boldsymbol{x}^{\prime}, \boldsymbol{y}^{\prime}, t\right)\right]^{+} \boldsymbol{G}^{T}\left(\boldsymbol{y}, t ; \boldsymbol{y}^{\prime}, t_{0}\right) d \boldsymbol{x}^{\prime} d \boldsymbol{y}^{\prime} \\
& +\int_{D^{2}} \boldsymbol{G}\left(\boldsymbol{x}, t ; \boldsymbol{x}^{\prime}, t_{0}\right)\left[\boldsymbol{P}_{0}^{+}\left(\boldsymbol{x}^{\prime}, \boldsymbol{y}^{\prime}\right)+\boldsymbol{M}\left(\boldsymbol{x}^{\prime}, \boldsymbol{y}^{\prime}, t\right)\right]^{+} \frac{\partial \boldsymbol{G}^{T}\left(\boldsymbol{y}, t ; \boldsymbol{y}^{\prime}, t_{0}\right)}{\partial t} d \boldsymbol{x}^{\prime} d \boldsymbol{y}^{\prime}+\Omega \\
= & \int_{D^{2}} \overline{\boldsymbol{A}}_{\boldsymbol{x}} \boldsymbol{G}\left(\boldsymbol{x}, t ; \boldsymbol{x}^{\prime}, t_{0}\right)\left[\boldsymbol{P}_{0}^{+}\left(\boldsymbol{x}^{\prime}, \boldsymbol{y}^{\prime}\right)+\boldsymbol{M}\left(\boldsymbol{x}^{\prime}, \boldsymbol{y}^{\prime}, t\right)\right]^{+} \boldsymbol{G}^{T}\left(\boldsymbol{y}, t ; \boldsymbol{y}^{\prime}, t_{0}\right) d \boldsymbol{x}^{\prime} d \boldsymbol{y}^{\prime} \\
& +\int_{D^{2}} \boldsymbol{G}\left(\boldsymbol{x}, t ; \boldsymbol{x}^{\prime}, t_{0}\right)\left[\boldsymbol{P}_{0}^{+}\left(\boldsymbol{x}^{\prime}, \boldsymbol{y}^{\prime}\right)+\boldsymbol{M}\left(\boldsymbol{x}^{\prime}, \boldsymbol{y}^{\prime}, t\right)\right]^{+}\left[\overline{\boldsymbol{A}}_{\boldsymbol{y}} \boldsymbol{G}\left(\boldsymbol{y}, t ; \boldsymbol{y}^{\prime}, t_{0}\right)\right]^{T} d \boldsymbol{x}^{\prime} d \boldsymbol{y}^{\prime}+\Omega \\
= & \overline{\boldsymbol{A}}_{\boldsymbol{x}} \boldsymbol{P}(\boldsymbol{x}, \boldsymbol{y}, t)+\boldsymbol{P}(\boldsymbol{x}, \boldsymbol{y}, t)\left[\overline{\boldsymbol{A}}_{\boldsymbol{y}}\right]^{T}+\Omega,
\end{aligned}
$$

where we have used the first equation of (23), and

$$
\Omega=\int_{D^{2}} \boldsymbol{G}\left(\boldsymbol{x}, t ; \boldsymbol{x}^{\prime}, t_{0}\right) \frac{\partial\left[\boldsymbol{P}_{0}^{+}\left(\boldsymbol{x}^{\prime}, \boldsymbol{y}^{\prime}\right)+\boldsymbol{M}\left(\boldsymbol{x}^{\prime}, \boldsymbol{y}^{\prime}, t\right)\right]^{+}}{\partial t} \boldsymbol{G}^{T}\left(\boldsymbol{y}, t ; \boldsymbol{y}^{\prime}, t_{0}\right) d \boldsymbol{x}^{\prime} d \boldsymbol{y}^{\prime} .
$$

Applying Lemma 3.2 to (81) arrives at

$$
\begin{aligned}
\Omega= & -\int_{D^{4}} \boldsymbol{G}\left(\boldsymbol{x}, t ; \boldsymbol{x}^{\prime}, t_{0}\right)\left[\boldsymbol{P}_{0}^{+}\left(\boldsymbol{x}^{\prime}, \boldsymbol{x}^{\prime \prime}\right)+\boldsymbol{M}\left(\boldsymbol{x}^{\prime}, \boldsymbol{x}^{\prime \prime}, t\right)\right]^{+} \frac{\partial \boldsymbol{M}\left(\boldsymbol{x}^{\prime \prime}, \boldsymbol{y}^{\prime \prime}, t\right)}{\partial t} \times \\
& {\left[\boldsymbol{P}_{0}^{+}\left(\boldsymbol{y}^{\prime \prime}, \boldsymbol{y}^{\prime}\right)+\boldsymbol{M}\left(\boldsymbol{y}^{\prime \prime}, \boldsymbol{y}^{\prime}, t\right)\right]^{+} \boldsymbol{G}^{T}\left(\boldsymbol{y}, t ; \boldsymbol{y}^{\prime}, t_{0}\right) d \boldsymbol{x}^{\prime} d \boldsymbol{y}^{\prime} d \boldsymbol{x}^{\prime \prime} d \boldsymbol{y}^{\prime \prime} }
\end{aligned}
$$

Using the expression of $\boldsymbol{M}(\boldsymbol{x}, \boldsymbol{y}, t)$ defined in (22) gives

$$
\begin{aligned}
\frac{\partial \boldsymbol{M}\left(\boldsymbol{x}^{\prime \prime}, \boldsymbol{y}^{\prime \prime}, t\right)}{\partial t} & =\int_{D^{2}} \boldsymbol{G}^{T}\left(\boldsymbol{\eta}, t ; \boldsymbol{x}, t_{0}\right) \overline{\boldsymbol{R}}_{d}(\boldsymbol{\eta}, \boldsymbol{\alpha}, t) \boldsymbol{G}\left(\boldsymbol{\alpha}, t ; \boldsymbol{y}, t_{0}\right) d \boldsymbol{\eta} d \boldsymbol{\alpha} \\
& +\int_{S^{2}} \boldsymbol{G}^{T}\left(\boldsymbol{\xi}, t ; \boldsymbol{x}, t_{0}\right) \overline{\boldsymbol{R}}_{b}(\boldsymbol{\xi}, \boldsymbol{\gamma}, t) \boldsymbol{G}\left(\boldsymbol{\gamma}, t ; \boldsymbol{y}, t_{0}\right) d S_{\boldsymbol{\xi}} d S_{\boldsymbol{\gamma}}
\end{aligned}
$$


which is substituted into (82) yields

$$
\begin{aligned}
\Omega & =-\int_{D^{6}} \boldsymbol{G}\left(\boldsymbol{x}, t ; \boldsymbol{x}^{\prime}, t_{0}\right)\left[\boldsymbol{P}_{0}^{+}\left(\boldsymbol{x}^{\prime}, \boldsymbol{x}^{\prime \prime}\right)+\boldsymbol{M}\left(\boldsymbol{x}^{\prime}, \boldsymbol{x}^{\prime \prime}, t\right)\right]^{+} \boldsymbol{G}^{T}\left(\boldsymbol{\eta}, t ; \boldsymbol{x}, t_{0}\right) \overline{\boldsymbol{R}}_{d}(\boldsymbol{\eta}, \boldsymbol{\alpha}, t) \\
& \times \boldsymbol{G}\left(\boldsymbol{\alpha}, t ; \boldsymbol{y}, t_{0}\right)\left[\boldsymbol{P}_{0}^{+}\left(\boldsymbol{y}^{\prime \prime}, \boldsymbol{y}^{\prime}\right)+\boldsymbol{M}\left(\boldsymbol{y}^{\prime \prime}, \boldsymbol{y}^{\prime}, t\right)\right]^{+} \boldsymbol{G}^{T}\left(\boldsymbol{y}, t ; \boldsymbol{y}^{\prime}, t_{0}\right) d \boldsymbol{x}^{\prime} d \boldsymbol{y}^{\prime} d \boldsymbol{x}^{\prime \prime} d \boldsymbol{y}^{\prime \prime} d \boldsymbol{\eta} d \boldsymbol{\alpha} \\
& -\int_{D^{4}} \int_{S^{2}} \boldsymbol{G}\left(\boldsymbol{x}, t ; \boldsymbol{x}^{\prime}, t_{0}\right)\left[\boldsymbol{P}_{0}^{+}\left(\boldsymbol{x}^{\prime}, \boldsymbol{x}^{\prime \prime}\right)+\boldsymbol{M}\left(\boldsymbol{x}^{\prime}, \boldsymbol{x}^{\prime \prime}, t\right)\right]^{+} \boldsymbol{G}^{T}\left(\boldsymbol{\xi}, t ; \boldsymbol{x}, t_{0}\right) \overline{\boldsymbol{R}}_{b}(\boldsymbol{\xi}, \boldsymbol{\gamma}, t) \\
& \times \boldsymbol{G}\left(\boldsymbol{\gamma}, t ; \boldsymbol{y}, t_{0}\right)\left[\boldsymbol{P}_{0}^{+}\left(\boldsymbol{y}^{\prime \prime}, \boldsymbol{y}^{\prime}\right)+\boldsymbol{M}\left(\boldsymbol{y}^{\prime \prime}, \boldsymbol{y}^{\prime}, \bar{t}\right)\right]^{+} \boldsymbol{G}^{T}\left(\boldsymbol{y}, t ; \boldsymbol{y}^{\prime}, t_{0}\right) d \boldsymbol{x}^{\prime} d \boldsymbol{y}^{\prime} d \boldsymbol{x}^{\prime \prime} d \boldsymbol{y}^{\prime \prime} d S_{\boldsymbol{\xi}} d S_{\boldsymbol{\gamma}}
\end{aligned}
$$

Applying the first equation of (20) to the right hand side of (84) yields

$$
\begin{aligned}
\Omega= & -\int_{D^{2}} \boldsymbol{P}(\boldsymbol{x}, \boldsymbol{\eta}, t) \overline{\boldsymbol{R}}_{d}(\boldsymbol{\eta}, \boldsymbol{\alpha}, t) \boldsymbol{P}(\boldsymbol{\alpha}, \boldsymbol{y}, t) d \boldsymbol{\eta} d \boldsymbol{\alpha} \\
& -\int_{S^{2}} \boldsymbol{P}(\boldsymbol{x}, \boldsymbol{\xi}, t) \overline{\boldsymbol{R}}_{b}(\boldsymbol{\xi}, \boldsymbol{\gamma}, t) \boldsymbol{P}(\boldsymbol{\gamma}, \boldsymbol{y}, t) d S_{\boldsymbol{\xi}} d S_{\boldsymbol{\gamma}}
\end{aligned}
$$

Substituting (85) into (80) yields the first equation of (20).

\section{D.2 Proof of uniqueness of $\boldsymbol{P}(\boldsymbol{x}, \boldsymbol{y}, t)$}

It is seen from (20) that a trivial solution is $\boldsymbol{P}(\boldsymbol{x}, \boldsymbol{y}, t)=0$ for all $(\boldsymbol{x}, \boldsymbol{y}) \in D \times D$ and $t \geq t_{0}$. Moreover, since $\boldsymbol{P}\left(\boldsymbol{x}, \boldsymbol{y}, t_{0}\right)=\boldsymbol{P}_{0}(\boldsymbol{x}, \boldsymbol{y})$ is symmetric and positive definite, we focus on a non-zero solution of $(20)$. As such, we let $\boldsymbol{P}^{+}(\boldsymbol{x}, \boldsymbol{y}, t)$ be the generalized inverse of $\boldsymbol{P}(\boldsymbol{x}, \boldsymbol{y}, t)$. An application of Lemma 3.2 results in differentiation of $\boldsymbol{P}(\boldsymbol{x}, \boldsymbol{y}, t)$ with respect to $t$ along the solutions of $(20)$ :

$$
\begin{aligned}
& \frac{\partial \boldsymbol{P}^{+}(\boldsymbol{x}, \boldsymbol{y}, t)}{\partial t}=-\int_{D^{2}} \boldsymbol{P}^{+}\left(\boldsymbol{x}, \boldsymbol{x}^{\prime}, t\right) \frac{\partial \boldsymbol{P}\left(\boldsymbol{x}^{\prime}, \boldsymbol{y}^{\prime}, t\right)}{\partial t} \boldsymbol{P}^{+}\left(\boldsymbol{y}^{\prime}, \boldsymbol{y}, t\right) d \boldsymbol{x}^{\prime} d \boldsymbol{y}^{\prime} \\
& =-\int_{D^{2}} \boldsymbol{P}^{+}\left(\boldsymbol{x}, \boldsymbol{x}^{\prime}, t\right) \overline{\boldsymbol{A}}_{\boldsymbol{x}^{\prime}} \boldsymbol{P}\left(\boldsymbol{x}^{\prime}, \boldsymbol{y}^{\prime}, t\right) \boldsymbol{P}^{+}\left(\boldsymbol{y}^{\prime}, \boldsymbol{y}, t\right) d \boldsymbol{x}^{\prime} d \boldsymbol{y}^{\prime} \\
& -\int_{D^{2}} \boldsymbol{P}^{+}\left(\boldsymbol{x}, \boldsymbol{x}^{\prime}, t\right) \boldsymbol{P}\left(\boldsymbol{x}^{\prime}, \boldsymbol{y}^{\prime}, t\right)\left[\overline{\boldsymbol{A}}_{\boldsymbol{y}^{\prime}}\right]^{T} \boldsymbol{P}^{+}\left(\boldsymbol{y}^{\prime}, \boldsymbol{y}, t\right) d \boldsymbol{x}^{\prime} d \boldsymbol{y}^{\prime} \\
& +\int_{D^{4}} \boldsymbol{P}^{+}\left(\boldsymbol{x}, \boldsymbol{x}^{\prime}, t\right) \boldsymbol{P}\left(\boldsymbol{x}^{\prime}, \boldsymbol{\eta}, t\right) \overline{\boldsymbol{R}}_{d}(\boldsymbol{\eta}, \boldsymbol{\alpha}, t) \boldsymbol{P}\left(\boldsymbol{\alpha}, \boldsymbol{y}^{\prime}, t\right) \boldsymbol{P}^{+}\left(\boldsymbol{y}^{\prime}, \boldsymbol{y}, t\right) d \boldsymbol{x}^{\prime} d \boldsymbol{y}^{\prime} d \boldsymbol{\eta} d \boldsymbol{\alpha} \\
& +\int_{D^{2}} \int_{S^{2}} \boldsymbol{P}^{+}\left(\boldsymbol{x}, \boldsymbol{x}^{\prime}, t\right) \boldsymbol{P}\left(\boldsymbol{x}^{\prime}, \boldsymbol{\xi}, \bar{t}\right) \overline{\boldsymbol{R}}_{b}(\boldsymbol{\xi}, \boldsymbol{\gamma}, \bar{t}) \overline{\boldsymbol{P}}\left(\boldsymbol{\gamma}, \boldsymbol{y}^{\prime}, t\right) \boldsymbol{P}^{+}\left(\boldsymbol{y}^{\prime}, \boldsymbol{y}, t\right) d \boldsymbol{x}^{\prime} d \boldsymbol{y}^{\prime} d S_{\boldsymbol{\xi}} d S_{\boldsymbol{\gamma}} \\
& =-\int_{D} \boldsymbol{P}^{+}\left(\boldsymbol{x}, \boldsymbol{x}^{\prime}, t\right) \overline{\boldsymbol{A}}_{\boldsymbol{x}^{\prime}} \boldsymbol{I} \delta\left(\boldsymbol{x}^{\prime}-\boldsymbol{y}\right) d \boldsymbol{x}^{\prime}-\int_{D} \boldsymbol{I} \delta\left(\boldsymbol{x}-\boldsymbol{y}^{\prime}\right) \boldsymbol{y}^{\prime}\left[\overline{\boldsymbol{A}}_{\boldsymbol{y}^{\prime}}\right]^{T} \boldsymbol{P}^{+}\left(\boldsymbol{y}^{\prime}, \boldsymbol{y}, t\right) d \boldsymbol{y}^{\prime} \\
& +\int_{D^{2}} \boldsymbol{I}(\boldsymbol{x}-\boldsymbol{\eta}) \overline{\boldsymbol{R}}_{d}(\boldsymbol{\eta}, \boldsymbol{\alpha}, \bar{t}) \boldsymbol{I} \delta(\boldsymbol{\alpha}-\boldsymbol{y}) \boldsymbol{\eta} d \boldsymbol{\alpha}+\int_{S^{2}} \boldsymbol{I} \delta(\boldsymbol{x}-\boldsymbol{\xi}) \overline{\boldsymbol{R}}_{b}(\boldsymbol{\xi}, \boldsymbol{\gamma}, t) \boldsymbol{I} \delta(\boldsymbol{\gamma}-\boldsymbol{y}) d S_{\boldsymbol{\xi}} d S_{\boldsymbol{\gamma}}, \\
& =-\boldsymbol{P}^{+}(\boldsymbol{x}, \boldsymbol{y}, t) \overline{\boldsymbol{A}}_{\boldsymbol{y}}-\left[\overline{\boldsymbol{A}}_{\boldsymbol{x}}\right]^{T} \boldsymbol{P}^{+}(\boldsymbol{x}, \boldsymbol{y}, t)+\overline{\boldsymbol{R}}_{d}(\boldsymbol{x}, \boldsymbol{y}, t)+\overline{\boldsymbol{R}}_{b}(\boldsymbol{\xi}, \boldsymbol{\gamma}, t) .
\end{aligned}
$$


An application of Lemma 3.3 to the last equation of (86) shows that there exists a unique solution $\boldsymbol{P}^{+}(\boldsymbol{x}, \boldsymbol{y}, t)$, which is given in the third equation of (21). Finally, boundedness and positive definiteness of the unique solution $\boldsymbol{P}^{+}(\boldsymbol{x}, \boldsymbol{y}, t)$ follow from the item 3) in Assumption 2.1 and (86) since $\overline{\boldsymbol{R}}_{d}(\boldsymbol{x}, \boldsymbol{y}, t)$ and $\overline{\boldsymbol{R}}_{b}(\boldsymbol{\xi}, \boldsymbol{\gamma}, t)$ are bounded by the item 2) of Assumption 2.1 and are symmetric and positive definite.

\section{E Derivation of (28)}

Let us introduce an Euler or weak variation $\delta \boldsymbol{\chi}(\boldsymbol{x}, t)=\varepsilon \boldsymbol{\varrho}(\boldsymbol{x}, t)$, where $\boldsymbol{\varrho}(\boldsymbol{x}, t)$ is an arbitrary twice continuously differentiable function. The necessary conditions for the cost functional $J_{1}$ to be minimized are found by setting the weak variation $\delta J_{1}$ of the functional $J_{1}$ to be equal to zero. Now assuming that the end points $t_{0}$ and $t_{f}$ are not fixed so as when the parameter $\varepsilon$ varies so do the end points $t_{0}$ and $t_{f}$, i.e., $t_{0}=t_{0}(\varepsilon)$ and $t_{f}=t_{f}(\varepsilon)$. As such, the weak variation $\delta J_{1}$ is given by

$$
\begin{aligned}
\delta J_{1} & =\left.\left[\frac{\partial}{\partial \varepsilon} \int_{t_{0}(\varepsilon)}^{t_{f}(\varepsilon)} L_{1} d t\right]\right|_{\varepsilon \rightarrow 0}+\delta J_{0} \\
& =\left.\left[\left.L_{1}\right|_{t=t_{f}} \frac{d t_{f}(\varepsilon)}{d \varepsilon}\right]\right|_{\varepsilon \rightarrow 0}-\left.\left[\left.L_{1}\right|_{t=t_{0}} \frac{d t_{0}(\varepsilon)}{d \varepsilon}\right]\right|_{\varepsilon \rightarrow 0}+\left.\int_{t_{0}(\varepsilon)}^{t_{f}(\varepsilon)}\left[\frac{\partial L_{1}}{\partial \varepsilon}\right]\right|_{\varepsilon \rightarrow 0} d t+\delta J_{0},
\end{aligned}
$$

where we have used Leibnitz's differentiation rule. On the other hand, a calculation shows that

$$
\begin{aligned}
& {\left.\left[\left.L_{1}\right|_{t=t_{f}} \frac{d t_{f}(\varepsilon)}{d \varepsilon}\right]\right|_{\varepsilon \rightarrow 0}-\left.\left[\left.L_{1}\right|_{t=t_{0}} \frac{d t_{0}(\varepsilon)}{d \varepsilon}\right]\right|_{\varepsilon \rightarrow 0}=\left.L_{1} \delta t\right|_{t_{0}} ^{t_{f}},} \\
& \left.\int_{t_{0}(\varepsilon)}^{t_{f}(\varepsilon)}\left[\frac{\partial L_{1}}{\partial \varepsilon}\right]\right|_{\varepsilon \rightarrow 0} d t=\int_{t_{0}}^{t_{f}} \int_{D}\left\langle\frac{\delta L_{1}}{\delta \boldsymbol{\chi}(\boldsymbol{x}, t)}-\frac{\partial}{\partial t}\left[\frac{\delta L_{1}}{\delta(\partial \boldsymbol{\chi}(\boldsymbol{x}, t) / \partial t)}\right], \boldsymbol{\varrho}(\boldsymbol{x}, t)\right\rangle d \boldsymbol{x} d t \\
& \quad+\int_{S}\left\langle\frac{\delta L_{1}}{\delta \boldsymbol{\chi}(\boldsymbol{\xi}, t)}, \boldsymbol{\varrho}(\boldsymbol{\xi}, t)\right\rangle d S_{\boldsymbol{\xi}}-\left.\left[\int_{D}\left\langle\frac{\delta L_{1}}{\delta(\partial \boldsymbol{\chi}(\boldsymbol{x}, t) / \partial t)}, \frac{\partial \boldsymbol{\chi}(\boldsymbol{x}, t)}{\partial t}\right\rangle d \boldsymbol{x}\right] \delta t\right|_{t_{0}} ^{t_{f}} \\
& \quad+\left.\left[\int_{D}\left\langle\frac{\delta L_{1}}{\delta(\partial \boldsymbol{\chi}(\boldsymbol{x}, t) / \partial t)}, \delta \boldsymbol{\chi}(\boldsymbol{x}, t)\right\rangle d \boldsymbol{x}\right]\right|_{t_{0}} ^{t_{f}}, \\
& \delta J_{0}=\int_{D}\left\langle\frac{\delta J_{0}}{\delta \boldsymbol{\chi}\left(\boldsymbol{x}, t_{0}\right)}, \delta \boldsymbol{\chi}\left(\boldsymbol{x}, t_{0}\right)\right\rangle d \boldsymbol{x} .
\end{aligned}
$$

We calculate $\delta L_{1}$ as follows:

$$
\begin{aligned}
\delta L_{1}= & \left.\int_{D} \frac{\delta L_{1}}{\delta \varepsilon}\right|_{\varepsilon \rightarrow 0} d \boldsymbol{x}+\left.\int_{S} \frac{\delta L_{1}}{\delta \varepsilon}\right|_{\varepsilon \rightarrow 0} d S_{\boldsymbol{\xi}} \\
= & -\int_{D^{2}}\left\langle\boldsymbol{H}_{d}^{T}(\boldsymbol{x}, t) \boldsymbol{Q}_{d}^{+}(\boldsymbol{x}, \boldsymbol{y}, t)\left[\boldsymbol{z}_{d}(\boldsymbol{y}, t)-\boldsymbol{H}_{d}(\boldsymbol{y}, t) \boldsymbol{\chi}(\boldsymbol{y}, t)\right], \boldsymbol{\varrho}(\boldsymbol{x}, t)\right\rangle d \boldsymbol{x} d \boldsymbol{y} \\
& +\int_{D}\left\langle\boldsymbol{\lambda}(\boldsymbol{x}, t), \boldsymbol{A}_{\boldsymbol{x}} \boldsymbol{\varrho}(\boldsymbol{x}, t)\right\rangle d \boldsymbol{x}-\int_{S}\left\langle\boldsymbol{\lambda}(\boldsymbol{\xi}, t), \boldsymbol{\beta}_{\boldsymbol{\xi}} \boldsymbol{\varrho}(\boldsymbol{\xi}, t)\right\rangle d S_{\boldsymbol{\xi}} \\
& -\int_{S^{2}}\left\langle\boldsymbol{H}_{b}^{T}(\boldsymbol{\xi}, t) \boldsymbol{Q}_{b}^{+}(\boldsymbol{\xi}, \boldsymbol{\alpha}, t)\left[\boldsymbol{z}_{b}(\boldsymbol{\alpha}, t)-\boldsymbol{H}_{b}(\boldsymbol{\alpha}, t) \boldsymbol{\chi}(\boldsymbol{\alpha}, t), \varrho(\boldsymbol{\xi}, t)\right\rangle d S_{\boldsymbol{\xi}} d S_{\boldsymbol{\alpha}}\right.
\end{aligned}
$$


From Lemma 3.1, we have

$$
\begin{aligned}
\int_{D}\left\langle\boldsymbol{\lambda}(\boldsymbol{x}, t), \boldsymbol{A}_{\boldsymbol{x}} \boldsymbol{\varrho}(\boldsymbol{x}, t)\right\rangle d \boldsymbol{x}-\int_{S}\left\langle\boldsymbol{\beta}_{\boldsymbol{\xi}} \varrho(\boldsymbol{\xi}, t), \boldsymbol{\lambda}(\boldsymbol{\xi}, t)\right\rangle d S_{\boldsymbol{\xi}}= \\
\int_{D}\left\langle\boldsymbol{A}_{\boldsymbol{x}}^{*} \boldsymbol{\lambda}(\boldsymbol{x}, t), \varrho(\boldsymbol{x}, t)\right\rangle d \boldsymbol{x}-\int_{S}\left\langle\boldsymbol{\beta}_{\boldsymbol{\xi}}^{*} \boldsymbol{\lambda}(\boldsymbol{\xi}, t), \varrho(\boldsymbol{\xi}, t)\right\rangle d S_{\boldsymbol{\xi}}
\end{aligned}
$$

where $\boldsymbol{A}_{\boldsymbol{x}}^{*}$ and $\boldsymbol{\beta}_{\boldsymbol{\xi}}^{*}$ are given in (13). Substituting (90) into (89) results in

$$
\begin{aligned}
\delta L_{1} & =-\int_{D^{2}}\left\langle\boldsymbol{H}_{d}^{T}(\boldsymbol{x}, t) \boldsymbol{Q}_{d}^{+}(\boldsymbol{x}, \boldsymbol{y}, t)\left[\boldsymbol{z}_{d}(\boldsymbol{y}, t)-\boldsymbol{H}_{d}(\boldsymbol{y}, t) \boldsymbol{\chi}(\boldsymbol{y}, t)\right], \varrho(\boldsymbol{x}, t)\right\rangle d \boldsymbol{x} d \boldsymbol{y} \\
& +\int_{D}\left\langle\boldsymbol{A}_{\boldsymbol{x}}^{*} \boldsymbol{\lambda}(\boldsymbol{x}, t), \varrho(\boldsymbol{x}, t)\right\rangle d \boldsymbol{x}-\int_{S}\left\langle\boldsymbol{\beta}_{\boldsymbol{\xi}}^{*} \boldsymbol{\lambda}(\boldsymbol{\xi}, t), \varrho(\boldsymbol{\xi}, t)\right\rangle d S_{\boldsymbol{\xi}} \\
& -\int_{S^{2}}\left\langle\boldsymbol{H}_{b}^{T}(\boldsymbol{\xi}, t) \boldsymbol{Q}_{b}^{+}(\boldsymbol{\xi}, \boldsymbol{\alpha}, t)\left[\boldsymbol{z}_{b}(\boldsymbol{\alpha}, t)-\boldsymbol{H}_{b}(\boldsymbol{\alpha}, t) \boldsymbol{\chi}(\boldsymbol{\alpha}, t)\right], \varrho(\boldsymbol{\xi}, t)\right\rangle d S_{\boldsymbol{\xi}} d S_{\boldsymbol{\alpha}} .
\end{aligned}
$$

Thus, we have

$$
\begin{aligned}
& \frac{\delta L_{1}}{\delta \boldsymbol{\chi}(\boldsymbol{x}, t)}=\boldsymbol{A}_{\boldsymbol{x}}^{*} \boldsymbol{\lambda}(\boldsymbol{x}, t)-\int_{D} \boldsymbol{H}_{d}^{T}(\boldsymbol{x}, t) \boldsymbol{Q}_{d}^{+}(\boldsymbol{x}, \boldsymbol{y}, t)\left[\boldsymbol{z}_{d}(\boldsymbol{y}, t)-\boldsymbol{H}_{d}(\boldsymbol{y}, t) \boldsymbol{\chi}(\boldsymbol{y}, t)\right] d \boldsymbol{y}, \\
& \frac{\delta L_{1}}{\delta \boldsymbol{\chi}(\boldsymbol{\xi}, t)}=-\boldsymbol{\beta}_{\boldsymbol{\xi}}^{*} \boldsymbol{\lambda}(\boldsymbol{\xi}, t)-\int_{S} \boldsymbol{H}_{b}^{T}(\boldsymbol{\xi}, t) \boldsymbol{Q}_{b}^{+}(\boldsymbol{\xi}, \boldsymbol{\alpha}, t)\left[\boldsymbol{z}_{b}(\boldsymbol{\alpha}, t)-\boldsymbol{H}_{b}(\boldsymbol{\alpha}, t) \boldsymbol{\chi}(\boldsymbol{\alpha}, t)\right] d S_{\boldsymbol{\xi}} .
\end{aligned}
$$

As $\varrho(\boldsymbol{x}, t)$ is arbitrary and $\varrho\left(\boldsymbol{x}, t_{0}\right)=0$ and $\varrho\left(\boldsymbol{x}, t_{f}\right)=0$, setting $\delta J_{1}=0$ results in

$$
\begin{aligned}
& \frac{\delta L_{1}}{\delta \boldsymbol{\chi}(\boldsymbol{x}, t)}-\frac{\partial}{\partial t}\left[\frac{\delta L_{1}}{\delta(\partial \boldsymbol{\chi}(\boldsymbol{x}, t) / \partial t)}\right]=0, \frac{\delta L_{1}}{\delta \boldsymbol{\chi}(\boldsymbol{\xi}, t)}=0, \\
& {\left.\left[L_{1}-\int_{D}\left\langle\frac{\delta L_{1}}{\delta(\partial \boldsymbol{\chi}(\boldsymbol{x}, t) / \partial t)}, \frac{\partial \boldsymbol{\chi}(\boldsymbol{x}, t)}{\partial t}\right\rangle d \boldsymbol{x}\right] \delta t\right|_{t_{0}} ^{t_{f}}} \\
& +\left.\left[\int_{D}\left\langle\frac{\delta L_{1}}{\delta(\partial \boldsymbol{\chi}(\boldsymbol{x}, t) / \partial t)}, \delta \boldsymbol{\chi}(\boldsymbol{x}, t)\right\rangle d \boldsymbol{x}\right]\right|_{t_{0}} ^{t_{f}}+\int_{D}\left\langle\frac{\delta J_{0}}{\delta \boldsymbol{\chi}\left(\boldsymbol{x}, t_{0}\right)}, \delta \boldsymbol{\chi}\left(\boldsymbol{x}, t_{0}\right)\right\rangle d \boldsymbol{x}=0 .
\end{aligned}
$$

From the expressions of $L_{1}$ and $J_{0}$, see (27) and (11), and $t_{0}$ and $t_{f}$ are fixed for our current problem, the equations listed in (93) with the use of (92) are equivalent to the last four equations of (28). Similarly, we can derive the weak variation $\delta J_{1}$ with respect to $\boldsymbol{\lambda}(\boldsymbol{x}, t), \boldsymbol{u}_{d}(\boldsymbol{x}, t)$ and $\boldsymbol{u}_{b}(\boldsymbol{\xi}, t)$ to obtain

$$
\begin{aligned}
& \frac{\delta L_{1}}{\delta \boldsymbol{\lambda}(\boldsymbol{x}, t)}-\frac{\partial}{\partial t}\left[\frac{\delta L_{1}}{\delta(\partial \boldsymbol{\lambda}(\boldsymbol{x}, t) / \partial t)}\right]=0 \Rightarrow \frac{\partial \boldsymbol{\chi}(\boldsymbol{x}, t)}{\partial t}=\boldsymbol{A}_{\boldsymbol{x}} \boldsymbol{\chi}(\boldsymbol{x}, t)+\boldsymbol{u}_{d}(\boldsymbol{x}, t), \\
& \frac{\delta L_{1}}{\delta \boldsymbol{\lambda}(\boldsymbol{\xi}, t)}=0 \quad \Rightarrow \boldsymbol{\beta}_{\boldsymbol{\xi}} \boldsymbol{\chi}(\boldsymbol{\xi}, t)=\boldsymbol{u}_{b}(\boldsymbol{\xi}, t), \\
& \frac{\delta L_{1}}{\delta \boldsymbol{u}_{d}(\boldsymbol{x}, t)}-\frac{\partial}{\partial t}\left[\frac{\delta L_{1}}{\delta\left(\partial \boldsymbol{u}_{d}(\boldsymbol{x}, t) / \partial t\right)}\right]=0 \Rightarrow \boldsymbol{u}_{d}(\boldsymbol{x}, t)=-\int_{D} \boldsymbol{R}_{d}(\boldsymbol{x}, \boldsymbol{y}, t) \boldsymbol{\lambda}(\boldsymbol{y}, t) d \boldsymbol{y} \\
& \frac{\delta L_{1}}{\delta \boldsymbol{u}_{b}(\boldsymbol{\xi}, t)}-\frac{\partial}{\partial t}\left[\frac{\delta L_{1}}{\delta\left(\partial \boldsymbol{u}_{b}(\boldsymbol{\xi}, t) / \partial t\right)}\right]=0 \Rightarrow \boldsymbol{u}_{b}(\boldsymbol{\xi}, t)=-\int_{S} \boldsymbol{R}_{b}(\boldsymbol{\xi}, \boldsymbol{\alpha}, t) \boldsymbol{\lambda}(\boldsymbol{\alpha}, t) d S_{\boldsymbol{\alpha}},
\end{aligned}
$$

which are the first two equations of the set of EL equations (28). 


\section{References}

[1] B. D. O. Anderson and J. B. Moore, Optimal control: linear quadratic methods, Dover Publications, 1990.

[2] A. Bensoussan, G. Da Prato, M. C. Delfour, and S. K. Mitter, Representation and control of infinite dimensional systems, Birkhauser, Boston, MA, 2007.

[3] R. F. Curtain and H. J. Zwart, An introduction to infinite-dimensional linear systems theory, Springer-Verlag, New York, 1995.

[4] K.D. Do and J. Pan, Boundary control of transverse motion of marine risers with actuator dynamics, Journal of Sound and Vibration 318 (2008), no. 4-5, 768-791.

[5] __ Boundary control of three-dimensional inextensible marine risers, Journal of Sound and Vibration 327 (2009), no. 3-5, 299-321.

[6] W. Gawronski, Dynamics and control of structures a modal approach, SpringerVerlag, New York, 1998.

[7] L. M. Gelfand and G. E. Shirov, Generalized functions: Vol. 1, properties and operations, Academic Press, New York, 1964.

[8] H. Khalil, Nonlinear systems, Prentice Hall, 2002.

[9] M. Krstic and H. Deng, Stabilization of nonlinear uncertain systems, SpringerVerlag, London, 1998.

[10] M. Krstic, I. Kanellakopoulos, and P.V. Kokotovic, Nonlinear and adaptive control design, Wiley, New York, 1995.

[11] I. Lasiecka and R. Triggiani, Control theory for partial differential equations: Continuous and approximation theories, Cambridge University Press, Cambridge, UK, 2000.

[12] Z. H. Li and M. Krstic, Optimal design of adaptive tracking controllers for nonlinear systems, Automatica 33 (1997), 1459-1473.

[13] J.E. Marsden and A. J. Tromba, Vector calculus, 4th ed., W.H. Freeman and Company, N.Y, 1996.

[14] J. S. Meditch, Least squares filtering and smoothing for linear distributed parameter systems, Automatica (1971), 315-322.

[15] L. Meirovitch, Principles and techniques of vibrations, Prentice-Hall, 1997.

[16] T.L. Nguyen, K.D. Do, and J. Pan, Boundary control of two-dimensional marine risers with bending couplings, Journal of Sound and Vibration 332 (2013), no. 16, 3605-3622. 
[17] S. Ravindran, A reduced-order approach for optimal control of fluids using proper orthogonal decomposition, International Journal for Numerical Methods in Fluids 34 (2000), no. 5, 425-448.

[18] Y. Sakawa, Optimal filtering in linear distributed-parameter systems, International Journal of Engineering 16 (1972), 115-127.

[19] M. Thans, Towards a practical theory for distributed parameter systems, IEEE Transactions on Automatic Control 15 (1970), 245-247.

[20] F. E. Thau, On optimal filtering for a class of linear distributed parameter systems, Journal of Basic Engineering, Series D 91 (1969), 173-178.

[21] S. G. Tzafestas, On optimum distributed parameter filtering and fixed interval smoothing for coloured noise, IEEE Transactions on Automatic Control 17 (1972), 448-458.

[22] S. G. Tzafestas and J. M. Nightingale, Optimal control of a class of linear stochastic distributed-parameter systems, Proceedings of IEE 115 (1968), no. 8, $1213-1220$.

Received: July 3, 2013 